\title{
Antenna-coupled TES Bolometers used in BICEP2, Keck Array, and SPIDER
}

\section{Citation}

Ade, P. A. R., R. W. Aikin, M. Amiri, D. Barkats, S. J. Benton, C. A. Bischoff, J. J. Bock, et al. 2015. "Antenna-coupled TES Bolometers used in BICEP2, Keck Array, and SPIDER." The Astrophysical Journal 812 (2) (October 21): 176. doi:10.1088/0004-637x/812/2/176.

\section{Published Version}

doi:10.1088/0004-637X/812/2/176

\section{Permanent link}

http://nrs.harvard.edu/urn-3:HUL.InstRepos:30168450

\section{Terms of Use}

This article was downloaded from Harvard University's DASH repository, and is made available under the terms and conditions applicable to Open Access Policy Articles, as set forth at http:// nrs.harvard.edu/urn-3:HUL.InstRepos:dash.current.terms-of-use\#OAP

\section{Share Your Story}

The Harvard community has made this article openly available.

Please share how this access benefits you. Submit a story.

\section{Accessibility}




\title{
ANTENNA-COUPLED TES BOLOMETERS USED IN BICEP2, Keck Array, AND SPIDER
}

\author{
P. A. R. Ade ${ }^{1}$, R. W. Aikin ${ }^{2}$, M. Amiri ${ }^{3}$, D. Barkats ${ }^{4}$, S. J. Benton ${ }^{5}$, C. A. Bischoff ${ }^{6}$, J. J. Bock ${ }^{2,7}$, J. A. Bonetti ${ }^{7}$, \\ J. A. Brevik ${ }^{2}$, I. Buder ${ }^{6}$, E. Bullock ${ }^{8}$, G. Chattopadhyay ${ }^{7}$, G. Davis ${ }^{3}$, P. K. Day ${ }^{7}$, C. D. Dowell ${ }^{7}$, L. Duband ${ }^{9}$, \\ J. P. Filippini ${ }^{2,10}$, S. Fliescher ${ }^{11}$, S. R. Golwala ${ }^{2}$, M. Halpern ${ }^{3}$, M. Hasselfield ${ }^{12}$, S. R. Hildebrandt ${ }^{2,7}$, G. C. Hilton $^{13}$, \\ V. Hristov ${ }^{2}$, H. Hui ${ }^{2}$, K. D. Irwin ${ }^{13,14,15}$, W. C. Jones ${ }^{12}$, K. S. Karkare ${ }^{6}$, J. P. Kaufman ${ }^{16}$, B. G. Keating ${ }^{16}$, S. Kefeli ${ }^{2}$, \\ S. A. Kernasovskiy ${ }^{14}$, J. M. Kovac ${ }^{6}$, C. L. Kuo ${ }^{13,14}$, H. G. LeDuc ${ }^{7}$, E. M. Leitch ${ }^{17}$, N. Llombart ${ }^{7}$, M. Lueker $^{2}$, P. Mason ${ }^{2}$, \\ K. Megerian ${ }^{7}$, L. Moncelsi ${ }^{2}$, C. B. Netterfield ${ }^{5}$, H. T. Nguyen ${ }^{7}$, R. O’Brient ${ }^{2,7}$, R. W. Ogburn IV ${ }^{14,15}$, A. Orlando ${ }^{12}$, \\ C. Pryke ${ }^{11}$, A. S. Rahlin ${ }^{12}$, C. D. Reintsema ${ }^{13}$, S. Richter ${ }^{6}$, M. C. Runyan ${ }^{2,7}$, R. Schwarz ${ }^{11}$, C. D. Sheehy ${ }^{17,18}$, \\ Z. K. Staniszewsili ${ }^{2}$, R. V. Sudiwala ${ }^{1}$, G. P. Teply ${ }^{2}$, J. E. Tolan ${ }^{14}$, A. Trangsrud ${ }^{2,7}$, R. S. Tucker ${ }^{2}$, A. D. Turner ${ }^{7}$, \\ A. G. VieregG ${ }^{17,18}$, A. Weber ${ }^{7}$, D. V. Wiebe ${ }^{3}$, P. Wilson ${ }^{7}$, C. L. Wong ${ }^{6}$, K. W. Yoon ${ }^{14,15}$, And J. ZMuidzinas ${ }^{2,7}$ \\ (For the Bicep2, Keck Array, and Spider Collaborations) \\ ${ }^{1}$ School of Physics and Astronomy, Cardiff University, Cardiff, CF24 3AA, UK \\ ${ }^{2}$ Department of Physics, California Institute of Technology, Pasadena, CA 91125, USA; rogero@ caltech.edu \\ ${ }^{3}$ Department of Physics and Astronomy, University of British Columbia, Vancouver, BC, Canada \\ ${ }^{4}$ Joint ALMA Observatory, ESO, Santiago, Chile \\ ${ }^{5}$ Department of Physics, University of Toronto, Toronto, ON, Canada \\ ${ }^{6}$ Harvard-Smithsonian Center for Astrophysics, 60 Garden Street MS 42, Cambridge, MA 02138, USA \\ 7 Jet Propulsion Laboratory, Pasadena, CA 91109, USA \\ ${ }^{8}$ Minnesota Institute for Astrophysics, University of Minnesota, Minneapolis, MN 55455, USA \\ ${ }^{9}$ Université Grenoble Alpes, CEA INAC-SBT, F-38000 Grenoble, France \\ ${ }^{10}$ Department of Physics, University of Illinois at Urbana-Champaign, Urbana, IL 61820, USA \\ ${ }_{11}$ Department of Physics, University of Minnesota, Minneapolis, MN 55455, USA \\ ${ }_{12}$ Department of Physics, Princeton University, Princeton, NJ 08544, USA \\ ${ }^{13}$ National Institute of Standards and Technology, Boulder, CO 80305, USA \\ ${ }^{14}$ Department of Physics, Stanford University, Stanford, CA 94305, USA \\ ${ }^{15}$ Kavli Institute for Particle Astrophysics and Cosmology, SLAC National Accelerator Laboratory, 2575 Sand Hill Rd, Menlo Park, CA 94025, USA \\ ${ }_{17}$ Department of Physics, University of California at San Diego, La Jolla, CA 92093, USA \\ ${ }^{17}$ Kavli Institute for Cosmological Physics, University of Chicago, Chicago, IL 60637, USA \\ ${ }^{18}$ Department of Physics, Enrico Fermi Institute, University of Chicago, Chicago, IL 60637, USA \\ Received 2015 March 2; accepted 2015 July 28; published 2015 October 20
}

\begin{abstract}
We have developed antenna-coupled transition-edge sensor bolometers for a wide range of cosmic microwave background (CMB) polarimetry experiments, including BICEP2, Keck Array, and the balloon borne SPIDER. These detectors have reached maturity and this paper reports on their design principles, overall performance, and key challenges associated with design and production. Our detector arrays repeatedly produce spectral bands with $20 \%-30 \%$ bandwidth at 95,150 , or $230 \mathrm{GHz}$. The integrated antenna arrays synthesize symmetric co-aligned beams with controlled side-lobe levels. Cross-polarized response on boresight is typically $\sim 0.5 \%$, consistent with cross-talk in our multiplexed readout system. End-to-end optical efficiencies in our cameras are routinely $35 \%$ or higher, with per detector sensitivities of NET $\sim 300 \mu \mathrm{K}_{\mathrm{CMB}} \sqrt{\mathrm{s}}$. Thanks to the scalability of this design, we have deployed 2560 detectors as 1280 matched pairs in Keck Array with a combined instantaneous sensitivity of $\sim 9 \mu \mathrm{K}_{\mathrm{CMB}} \sqrt{\mathrm{s}}$, as measured directly from CMB maps in the 2013 season. Similar arrays have recently flown in the SPIDER instrument, and development of this technology is ongoing.
\end{abstract}

Key words: cosmic background radiation - instrumentation: detectors - instrumentation: polarimeters methods: laboratory: solid state - techniques: polarimetric

\section{INTRODUCTION}

Cosmic microwave background (CMB) polarimetry is a key observable to further our understanding of cosmology in both the later and early universe. Degree-scale B-mode polarization can be used to constrain the tensor-scalar ratio $r$ and place limits on the energy scale and potential form of inflation (Kamionkowski et al. 1997; Zaldarriaga \& Seljak 1997). Arcminute B-mode measurements allow precise reconstruction of the gravitational lensing potential at later times, which in turn can constrain the neutrino masses and the dark energy equation of state (Kaplinghat et al. 2003). Precise measurements of E-mode polarization provide further cosmological information on the plasma physics at recombination. Very precise future lensing polarization maps could ultimately be used for deeper searches of inflationary polarization. Finally a future space mission has the potential to measure large-scale polarization to astrophysical limits, for precise tests of inflation. However, these ambitious scientific goals require high sensitivity cameras with exquisite control of systematic errors.

To meet this need, we have developed large arrays of dualpolarized antenna-coupled transition edge sensor (TES) bolometers. The key feature in our design is optical coupling through a planar antenna, allowing the entire design to be fabricated with scalable photolithographic techniques. This has allowed us to rapidly deploy arrays for BICEP2, Keck Array, and the balloon borne SPIDER, amounting to over 6000 detectors fielded as of this writing. We deployed BICEP2 for observing in 2010 (Bicep2 Collaboration II 2014), three Keck 
Array $150 \mathrm{GHz}$ cameras for observing in 2011, and five $150 \mathrm{GHz}$ cameras for Keck Array in 2012 (Kernasovskiy et al. 2012). In 2014, we replaced two Keck Array $150 \mathrm{GHz}$ cameras with $95 \mathrm{GHz}$ cameras, and in 2015 we replaced two more with $230 \mathrm{GHz}$ cameras (fielding an additional 1000 detectors). SPIDER recently conducted a long-duration flight from McMurdo Station in January, deploying an additional 2400 detectors (Fraisse et al. 2013).

This paper describes the design principles of these detectors, as well as their on-sky performance and some challenges associated with their production. We draw from our extensive experience with the aforementioned experiments, and describe the as-deployed performance of our devices.

The format of this paper is as follows. In Sections 2 and 3 we describe the cameras and detector element designs, elaborating beyond the material in the BICEP2 Instrument Paper (Bicep2 Collaboration II 2014). Section 4 describes the fabrication techniques used. To optimize our designs and recipes, we had to characterize the optical properties of our thin films for millimeter waves, which we describe in Section 5. Section 6 describes the antenna array beam synthesis as well as related processing and design challenges. Our high on-sky detector yield is only possible through control of detector properties across each tile, which we discuss in Section 7. Section 8 describes the attained camera sensitivity and we show rough agreement between those measurements and a noise model. Finally, Section 9 offers some concluding remarks and describes future endeavors.

\section{OVERVIEW}

Cameras. Because the detector arrays are coupled to experiments with refracting optics, we briefly summarize their architecture. The BICEP2, Keck Array, and SPIDER cameras are similar $f / 2.226 \mathrm{~cm}$ aperture telecentric refracting telescopes, which re-image the sky onto the focal plane with pairs of highdensity polyethylene lenses. BICEP2 and Keck Array contain teflon, nylon, and reflective metal-mesh edge-filters that filter incident radiation to maintain low thermal loading on the focal plane such that internal closed-cycle ${ }^{3} \mathrm{He} /{ }^{3} \mathrm{He} /{ }^{4} \mathrm{He}$ sorption fridges can cool the focal plane to $280 \mathrm{mK}$ (Duband \& Collaudin 1999). In principle, lower base temperatures (achieved, for example, by a dilution fridge) can allow lower detector noise levels. However, BICEP2 achieved photon-noise limited sensitivities at these temperatures, and in the interest of scaling up to the larger multi-camera Keck Array and SPIDER systems, we were not motivated to further cool our detectors. Due to loading constraints at float, the SPIDER cameras cannot employ lossy teflon filters and instead utilize metal-mesh filters. They still use a nylon filter on the cold side of $4 \mathrm{~K}$. SPIDER uses a $\sim 2 \mathrm{~K}$ pumped helium bath and single stage closed cycle ${ }^{3} \mathrm{He}$ fridges to chill its focal planes to $\sim 300 \mathrm{mK}$. SPIDER uses ultra-high-molecular-weight polyethylene windows in its low pressure flight environment, whereas BICEP2 and Keck Array use closed cell nitrogen filled Zotefoam (respectively Propozote PPA30 and Plastazote HD30). We refer the reader to the Instrument Paper for more BICEP2specific details (Bicep2 Collaboration II 2014).

Focal plane. Each focal plane (Figure 1) contains four detector tiles mounted to a common copper frame. Each tile is a square of silicon cut from a standard $100 \mathrm{~mm}$ wafer, typically $550 \mu \mathrm{m}$ thick. The $150 \mathrm{GHz}$ BICEP2, SPIDER, and Keck Array focal planes contain four tiles, each consisting of 64 dual orthogonally polarized detector pairs, providing 512 detectors per camera. The detectors' beams terminate on their camera's cold stop at $-15 \mathrm{~dB}$ of the main lobe with $1.8 f \lambda$ spacing. The $95 \mathrm{GHz}$ Keck Array cameras have tiles containing 36 orthogonally polarized detector pairs, and thus 288 detectors per camera. These detectors' beams terminate on their camera's stop at $-12 \mathrm{~dB}$ of the main lobe with $1.5 f \lambda$ spacing. Current $230 \mathrm{GHz}$ focal planes contain four tiles of 64 detector-pairs each in order to match a readout system designed for the $150 \mathrm{GHz}$ cameras; this samples the focal plane less efficiently (with 2.6f $\lambda$ spacing), which will be addressed with a future higher density focal plane design. The $230 \mathrm{GHz}$ detectors' beams are similar in size to the $150 \mathrm{GHz}$ beams, terminating on the stop at $-15 \mathrm{~dB}$.

We mount the tiles to the aforementioned gold-plated copper frame and thermally sink each with a few hundred gold wire bonds linking the frame to gold bond-pads that directly contact the silicon substrate of the tile. The antennas are patterned on the non-illuminated side of the wafer, so that light arrives at the antenna through its silicon substrate (see Section 3.1 for elaboration). A quartz anti-reflection tile is mounted on the illuminated side of the wafer, while the antenna side faces a superconducting niobium reflective back-short placed $\lambda / 4$ away. Testing of early BICEP2 prototype focal planes suggested that near the tile edge, antennas for one of the two polarizations may couple to the frame, yielding elliptical beams in the far field. We have mitigated this coupling in all deployed focal planes by keeping the edge detector pairs a few wavelengths away from the frame and by corrugating the frames with specifically chosen depth and impedance grooves, optimized with simulations performed in the CST Microwave Suite (Corp 2009a).

Polarized Detector Elements. Our detector design is entirely planar and does not require horns or other contacting optics. In each detector element, optical power couples to two co-located, orthogonally polarized planar antenna arrays, each composed of slot sub-radiators patterned in a superconducting niobium $(\mathrm{Nb})$ ground plane. All slots of a given orientation are coherently combined through a microstrip summing tree to synthesize a single equivalent antenna for that polarization orientation. Power from each antenna is passed through an onchip band-defining filter before being dissipated on a suspended bolometer island. A TES voltage biased into its superconducting-normal transition on that island detects variations in the power received by the antennas.

\section{DETECTOR DESIGN}

\subsection{Antenna Design}

The antenna slots in each detector must be spaced to Nyquist sample the focal plane surface to avoid grating lobes that would rapidly change the impedance with frequency (Kuo et al. 2008). The antenna pattern of each axis of an array is calculated from the $N$ elements per linear dimension spaced at distance $s$ as follows:

$$
\begin{aligned}
A(\theta) & =\sum_{m=-(N-1) / 2}^{(N-1) / 2} e^{-j 2 \pi \frac{m s \sqrt{\epsilon_{r}}}{\lambda_{o}} \sin (\theta)} \\
& =\frac{\sin \left(N \pi s \sqrt{\epsilon_{r}} \sin \theta / \lambda_{o}\right)}{\sin \left(\pi s \sqrt{\epsilon_{r}} \sin \theta / \lambda_{o}\right)}
\end{aligned}
$$



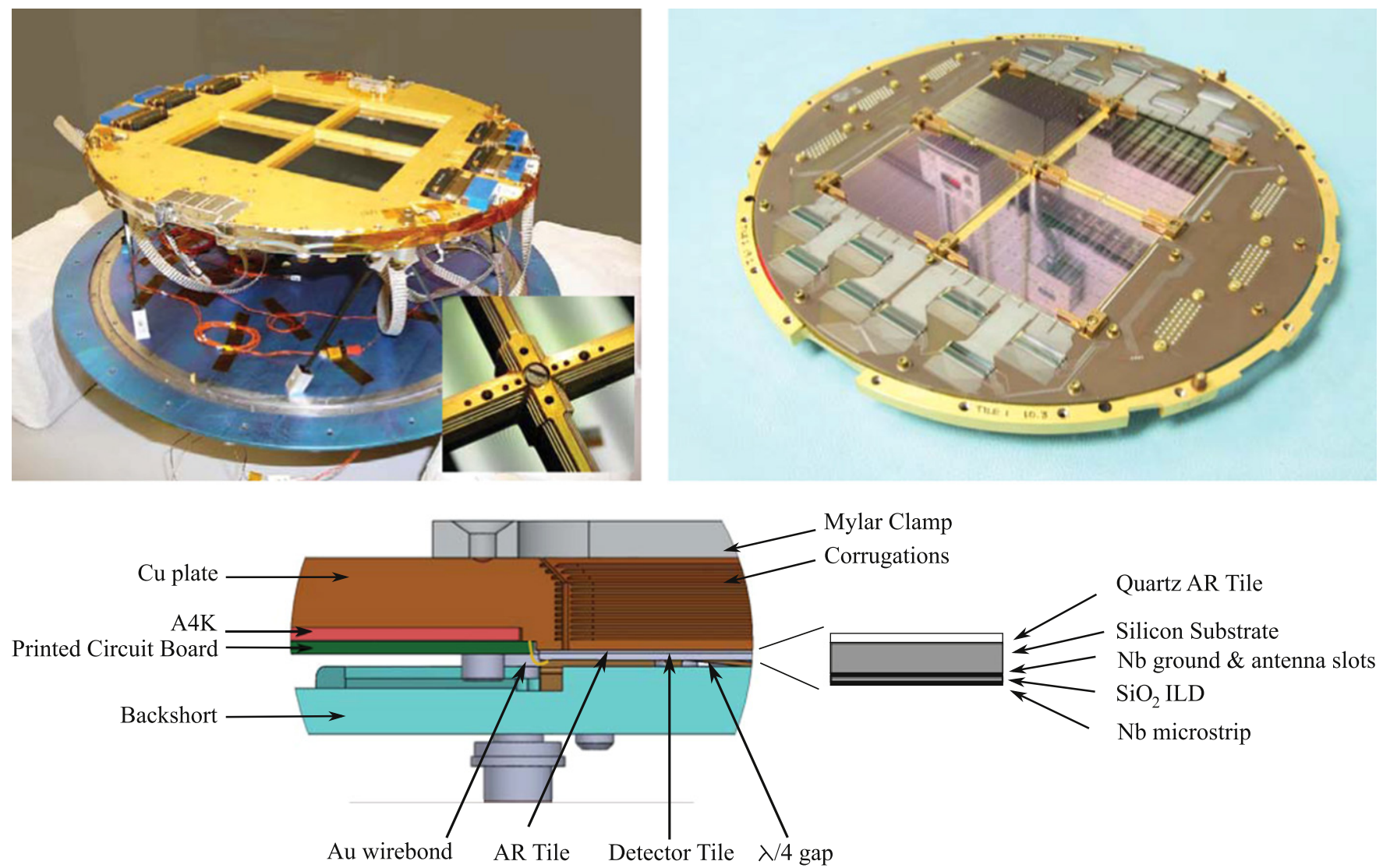

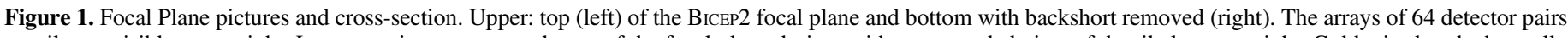

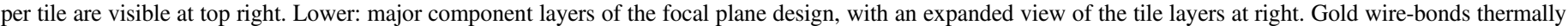

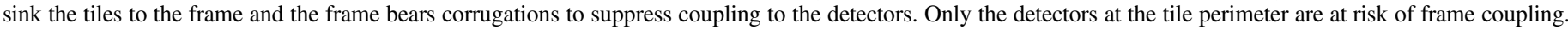
Corrugations are visible in the inset photo in the upper left panel.

where $\lambda_{o}$ is the free-space wavelength, $\epsilon_{r}$ the relative permittivity of the surrounding medium, and the sum is across sub-antennas indexed by $m$. In addition to the strong peak in the normal direction $(\theta=0)$, there are grating lobe peaks when $\sqrt{\epsilon_{r}} s \sin (\theta) / \lambda_{o}$ is a positive integer. To avoid these lobes, the slot spacing must be

$$
s \leqslant \frac{\lambda_{o, \min }}{\sqrt{\epsilon_{r}}}\left(1-\frac{1}{N}\right),
$$

where $\lambda_{o \text {, min }}$ is the minimum wavelength of operation and the term in parentheses accounts for the finite width of the grating-lobe peaks. For the $150 \mathrm{GHz}$ detectors fabricated on silicon $\left(\epsilon_{r}=11.8\right)$ with an upper band edge of $180 \mathrm{GHz}$ $\left(\lambda_{o, \min }=1.7 \mathrm{~mm}\right)$, the spacing must satisfy $s \leqslant 460 \mu \mathrm{m}$. To achieve this high density of radiators without intersection, we couple power to offset (echelon) pairs of radiators with a Bravais lattice defined in Figure 2. These dual-slot sub-arrays tile the detector elements in $8 \times 8,10 \times 10$, or $12 \times 12$ versions, where the overall detector size is chosen to match the $f / 2.2$ camera optics. By using such a large number of sub-antennas, we avoid excessive excitation of substrate modes that might degrade the detector's efficiency. The offset slot-pair geometry allows the two orthogonally polarized antenna arrays within each detector pair to be co-located (visible in upper two panels of Figure 2).

It is energetically favorable for the antennas to receive power through the silicon substrate to the vacuum side at a ratio of
$377 \Omega:\left|Z_{\mathrm{Si}}^{\text {in }}\right|$, where $Z_{\mathrm{Si}}^{\text {in }}$ (in Equation (4)) differs from silicon's TEM impedance of $110 \Omega$ in phase because standing waves in the substrate modify the effective impedance seen by the slots. We exploit this power difference by orienting the tiles with the silicon substrate side toward the sky and then terminating the back response on the vacuum side with a $\lambda / 4$ back-short. We mount $\lambda / 4$ quartz anti-reflection (AR) tiles to minimize reflection at the air-substrate interface.

\subsection{Antenna Impedance}

Optical power couples from the slots to a microstrip feed network. To minimize return loss at this interface, we need to accurately compute the input impedance of that feed network. We have written custom software that provides increased speed and versatility for this task over commercial options.

We compute the input impedance of our antenna from the required jump-discontinuity in magnetic fields across the slot at the microstrip feed-points $\left(J_{\text {feed }}=\Delta H_{x}\right.$ ) where we impose a driving current $J_{y}$. Modeling the currents in the ground plane with fictitious magnetic currents $\boldsymbol{M}=\hat{n} \times \boldsymbol{E}$ running longitudinally down the slot, the field jump-discontinuity requires:

$$
\begin{aligned}
J_{y}= & \int d x^{\prime} d y^{\prime} \boldsymbol{M}\left(x^{\prime}, y^{\prime}\right) \\
& \quad\left[G_{H_{1 x}, M_{x}}\left(x-x^{\prime}, y-y^{\prime}\right)-G_{H_{2 x}, M_{x}}\left(x-x^{\prime}, y-y^{\prime}\right)\right] .
\end{aligned}
$$



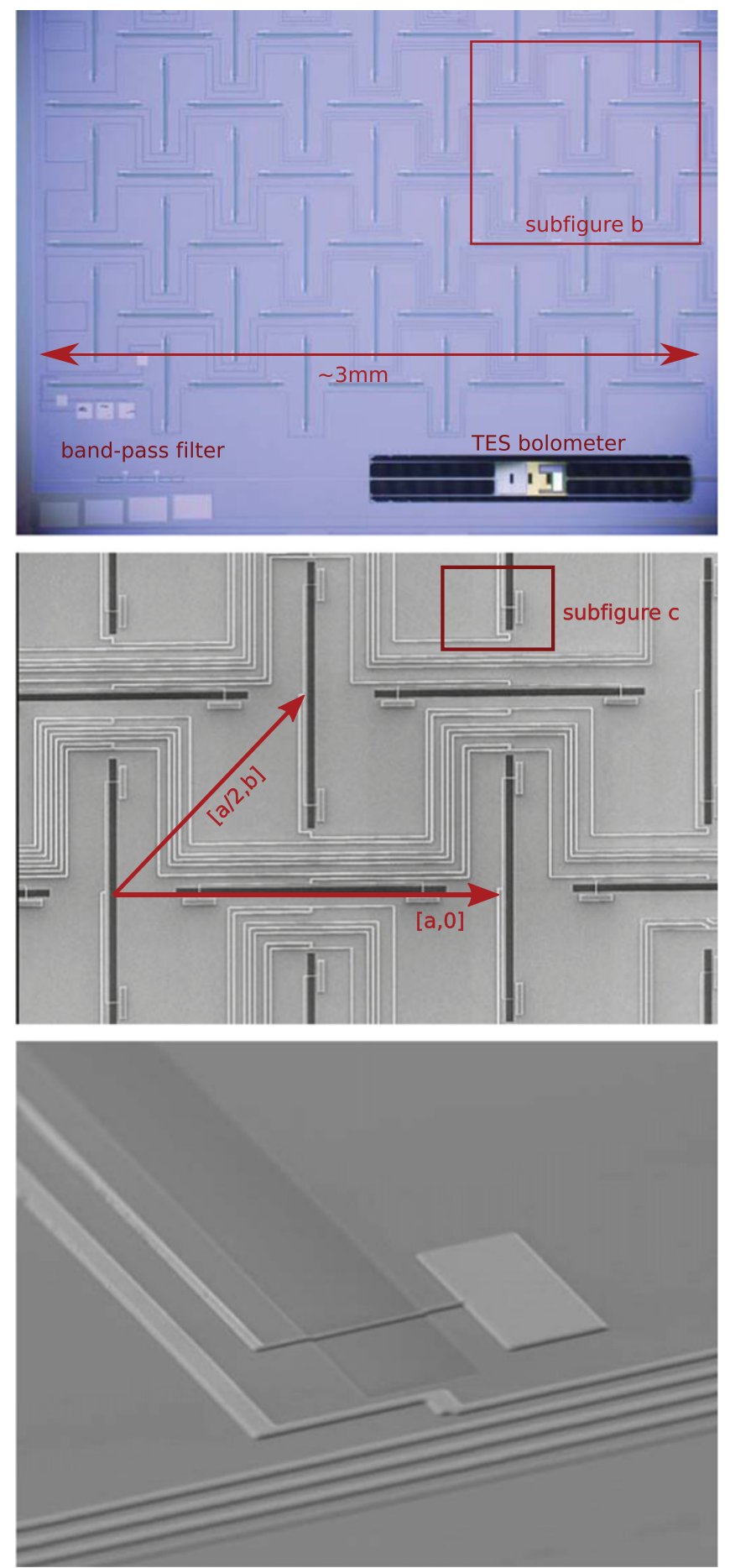

Figure 2. Optical and SEM photographs of major features of the antenna array. Upper: one quarter of a detector element. The antenna array, one filter, and one TES bolometer are visible, as well as DC readout lines for the detector. Middle: SEM micrograph of the slot array (dark rectangles) and oblique Bravais lattice (arrows). The thin white lines comprise the microstrip feed. For $150 \mathrm{GHz}$ detectors, $a \sim 600 \mu \mathrm{m}$ and $b=a / 2 \sim 300 \mu \mathrm{m}$; the slot dimensions and spacing in the $95 \mathrm{GHz}$ are $63 \%$ larger and those in the $230 \mathrm{GHz}$ detector elements are $47 \%$ smaller. Lower: SEM micrograph of microstrip crossover and shunt capacitor at a sub-antenna slot.

The antenna impedance can be inferred once these currents $\boldsymbol{M}$ are solved for. The Green functions $G_{H_{n}, M_{x}}$ describe the fields on either side of the slot $(n=[1,2]])$ resulting from an infinitesimal magnetic current in the $\boldsymbol{x}$ direction (parallel to the slot). In the spectral domain, these have the form

$$
\tilde{G}_{H_{x} M_{x}}\left(k_{x}, k_{y}, z=0\right)=\frac{\cos \left(\phi_{k}\right)}{Z_{\mathrm{TM}}^{\mathrm{in}}\left(k_{\rho}\right)}+\frac{\sin \left(\phi_{k}\right)}{Z_{\mathrm{TE}}^{\mathrm{in}}\left(k_{\rho}\right)}
$$

where $\phi_{k}=\arctan \left(k_{y} / k_{x}\right)$ and $k_{\rho}=\sqrt{k_{x}^{2}+k_{y}^{2}}$ (Das \& Pozar 1987). The dielectric films support transverse electric (TE) and transverse magnetic (TM) modes with different impedances. The impedance $Z_{\mathrm{TE}, \mathrm{TM}}^{\mathrm{in}}$ of each mode "seen" by the slots can be computed by solving for the standing waves of electric and magnetic fields in a manner analogous to treating the silicon substrate and quartz AR coating as transmission lines and then transforming the impedance of free space $(377 \Omega)$ through them (Das \& Pozar 1987). The backshort (ground) transforms through a quarter-wave space, presenting a parallel impedance that is open at the band center. The silicon substrates are a standard $100 \mathrm{~mm}$ diameter $550 \mu \mathrm{m}$ thick wafer, and we optimized the 95 and $150 \mathrm{GHz}$ impedance matching with this thickness in mind. The $230 \mathrm{GHz}$ versions are fabricated on thinner $370 \mu \mathrm{m}$ thick silicon substrates to scale this design.

We expand the unknown fictitious currents $M(x, y)=$ $\sum_{i} V_{i} F_{i}(x, y)$ in a one-dimensional piecewise sinusoidal basis (Kominami et al. 1985):

$$
\begin{aligned}
F_{i}(x, y)= & \frac{1}{\pi \sqrt{(w / 2)^{2}-y^{2}}} \\
& \times \begin{cases}\frac{\sin \left(k_{\rho}\left(\Delta x-\left|x-x_{i}\right|\right)\right)}{\sin \left(k_{\rho} \Delta x\right)}, & \left|x-x_{i}\right| \leqslant \Delta x \\
0, & \text { otherwise }\end{cases}
\end{aligned}
$$

localized in physical space at position $x_{i}$ over the slot, with sections of length $\Delta x$ and width $w$ corresponding to the slot width. This basis models the expected current distribution on the shorter slot dimensions, leaving the unknown distribution only on the longer dimension. Moreover the Fourier transform of the basis function has a compact analytic form. These features greatly simplify the computations. Solving Equation (3) with a Galerkin moment method reduces it to a matrix equation $\left[Y_{i j}\right]\left[V_{i}\right]=\left[I_{j}\right]$ (Kominami et al. 1985), where

$$
Y_{i j}=\int d k_{x} d k_{y} \tilde{F}_{i}^{*}\left(k_{x}, k_{y}\right) \tilde{G}\left(k_{x}, k_{y}, z=0\right) \tilde{F}_{j}\left(k_{x}, k_{y}\right) .
$$

The inverse of the admittance matrix in Equation (6) is an impedance between basis functions. The self and mutual impedance between basis functions located at feed-points is the input impedance that our feed-lines must match.

We avoid complicated numerical integration and further reduce the number of unknown $V_{i}$ s by assuming the arrays are infinite in extent and applying periodic boundary conditions where the antenna properties repeat for spectral translations along the lattice vectors:

$$
\begin{aligned}
k_{x}^{n} & =\frac{2 \pi n}{a} \\
k_{y}^{m, n} & =2 \pi\left(\frac{n}{a}+\frac{m}{b}\right)
\end{aligned}
$$

where $a$ and $b$ are the distances between the slots in the horizontal and vertical axes (see Figure 2). This approximation 


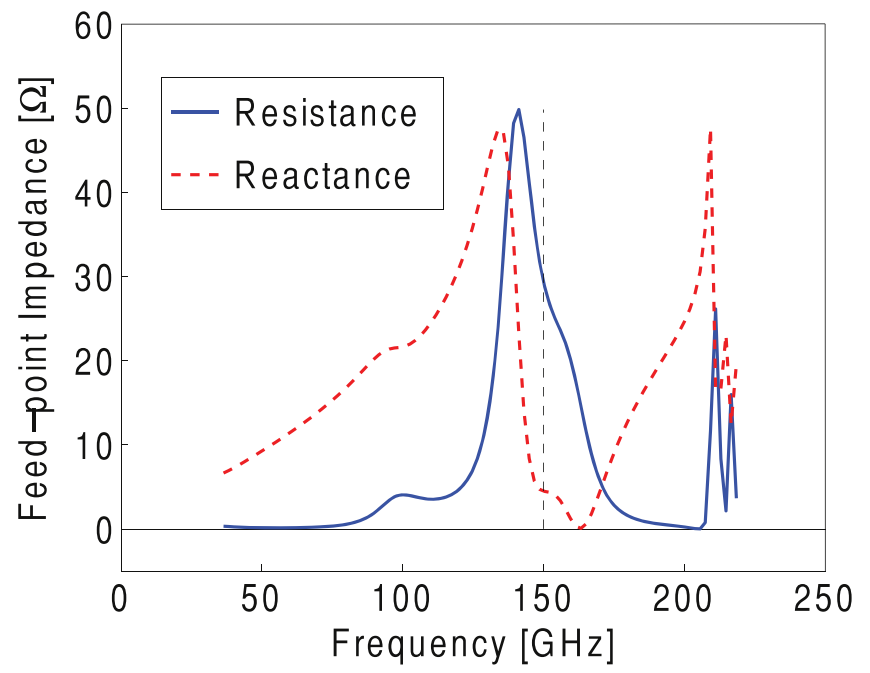

Figure 3. Simulated feed-point antenna impedance vs. frequency. The dashed vertical line is the band center.

is valid for slots in the interior of the array and reduces the integrals to a discrete sum that we terminate at a wavevector high enough to allow convergence. Figure 3 shows the computed input impedance against frequency for the $150 \mathrm{GHz}$ antennas. The oscillatory behavior at $200 \mathrm{GHz}$ is expected and real (i.e., not a numerical artifact) and coincides with the onset of grating lobes. We note that we have performed more numerically intensive simulations of finite slot arrays where continuous integration retains edge effects to some degree and these agree well with the infinite array approximation.

We couple power to microstrip lines at the points near the slot ends where the lines cross and shunt to the ground-plane on the opposite side (see lower panel of Figure 2). This coupling effectively transmutes the electric fields across the slot into fields between the microstrip conductor and ground plane, provided that impedances are well matched. To avoid grating lobes, we keep the slots as short as possible and excite the slots at their first resonance. However, the radiation resistance of center-fed slots at this resonance is nearly $300 \Omega$, caused by a current node at the slot center. By symmetrically feeding the slots with a pair of off-center microstrip lines close to the current anti-nodes, we greatly reduce the radiation resistance to $\sim 40 \Omega$. The microstrip feed lines must match this real antenna resistance, which, for our dielectric and metal film thicknesses, corresponds to the thinnest lines that we can reproducibly fabricate. The feed points also have a $5 \Omega$ inductive reactance, which is relatively stable over the band. We tune this away with series capacitors that shunt current to ground (also visible in Figure 2). Future designs may use more complicated terminations (e.g., open stubs) in lieu of capacitors to expand the bandwidth, allowing for co-located dual-color detectors.

\subsection{Microstrip Feed}

The waves from the slots coherently sum in the microstrip feed-network, which accomplishes beam synthesis in lieu of a horn. Figure 4 summarizes this topology. This network combines waves across rows, and then sums the waves from each row in a column tree at the side of the detector element, presenting a single microstrip line in each polarization. The two

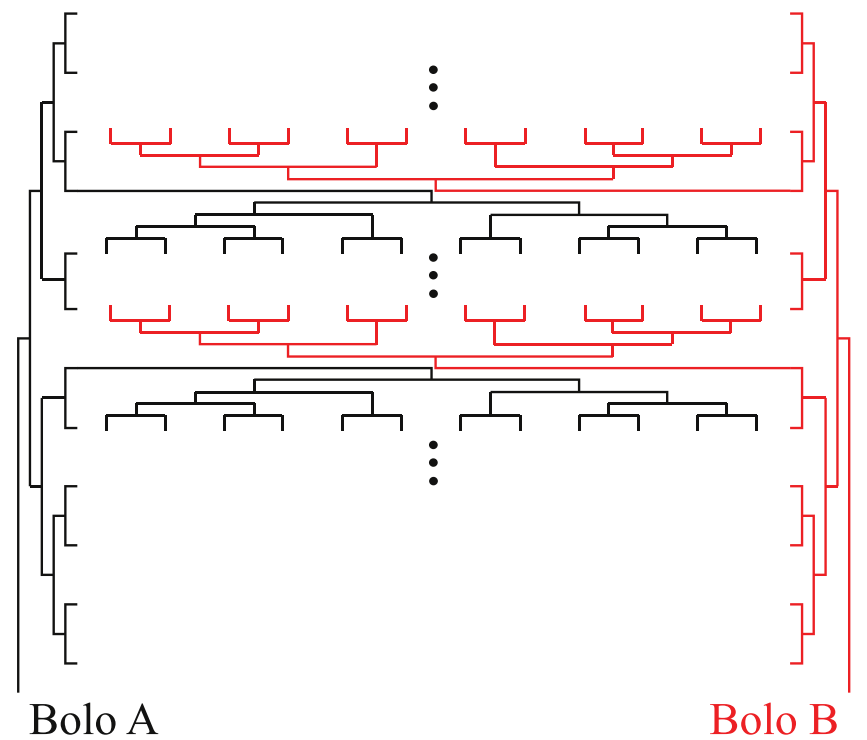

Figure 4. Abbreviated feed-network schematic for one detector pair, showing the branch structure for the two polarization summing trees.

orientations of slots couple power to two independent feeds that tightly interlock across the detector pair. The feeds are meant to be corporate, combining all waves with uniform phase, but the trees are not necessarily binary. In fact, the 230 and $150 \mathrm{GHz}$ detectors have a $12 \times 12$ array format while the $95 \mathrm{GHz}$ ones are $10 \times 10$, neither of which is a power of 2 .

Waves sum in microstrip tee-junctions, with impedances chosen to match across each junction when looking from the port closest to the bolometer. We pick the ratio of impedances on the ports closest to the slots to determine the illumination pattern. To accomplish this synthesis with low return loss, we must construct microstrips with correct impedances, which requires accurate knowledge of dielectric constants and the penetration depth into our superconducting niobium films. (See Section 5.) Thus far, all detectors deployed for BICEP2, Keck Array, and SPIDER produce a uniform top-hat illumination where the power splits are in proportion to the number of slots on each side of the junctions. The microstrip impedances can be chosen to synthesize an arbitrary pattern, however, and Gaussian tapered feeds have recently been deployed in the BICEP3 camera.

Coupling between microstrip lines can generate phase errors across the antennas, which we elaborate on in Section 6. The present designs have judiciously spaced the adjacent lines to minimize this coupling. To negate any residual phase errors, the feed-networks also contain sections of transmission line before each slot whose length we can vary during fabrication.

\subsection{Band-defining Filters}

Each microstrip feed contains an integrated band-defining filter between the antenna feed and bolometer. We use a threepole design as a compromise between bandwidth and loss: additional poles would let us increase the bandwidth and still avoid atmospheric features, but the additional passes of the waves through each resonator increase filter loss. Figure 5 shows a micrograph of the filter and a schematic of its equivalent circuit. We realize the resonators with lumped components, which do not suffer from the high frequency 


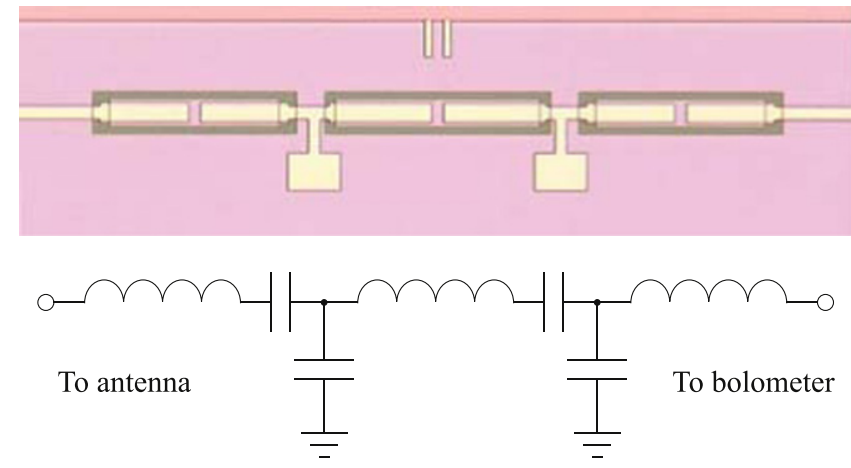

Figure 5. Microscope photograph of filter and equivalent circuit for $150 \mathrm{GHz}$.

resonant leaks present in $\lambda / 2$ and $\lambda / 4$ transmission line resonators.

Each pole is a series LC resonator, in which the inductors are short stretches of high-impedance coplanar waveguide (CPW). The CPW impedance $Z=\sqrt{L / C}$ of roughly $50 \Omega$ exceeds the surrounding lines and acts as a series inductor by allowing strong magnetic fields in the CPW ground-plane gaps. This inductance is almost entirely magnetic; kinetic inductance from the niobium superconducting microstrip and ground layers only makes a minor correction. The series capacitors are parallelplate metal-insulator-metal between upper and lower niobium films using the microstrip $\mathrm{SiO}_{2}$ as the dielectric. In an ideal design the central resonator would be a parallel LC resonator shunting to ground. For ease of fabrication we instead build a series resonator and invert its impedance on resonance using shunt capacitors to ground and reduced series capacitors (Galbraith \& Rebeiz 2008).

We numerically optimize the filter in simulations with the commercially available Sonnet software (Corp 2009b). The optimizations are constrained to maintain an entrance impedance of $10 \Omega$ which results in fabricable capacitors and inductors. Our design results in inductors longer than $\lambda / 8$ that have some slight parasitic shunt capacitance, but this does not degrade the performance appreciably. The filter in Figure 5 is tuned for a $150 \mathrm{GHz}$ band-center. Filters for 95 and $230 \mathrm{GHz}$ versions have components with the same reactance at the resonant frequencies. We show spectra and summarize spectral response features in Section 7.

\subsection{Bolometer Design}

We terminate and thermalize millimeter-wave power on a released bolometer island in a meandered lossy gold microstrip. $150 \mathrm{GHz}$ waves are constrained to propagate through a skin depth of $180 \mathrm{~nm}$, and over a length of several wavelengths the line absorbs $99 \%$ of the power between two passes. We scale the 230 and $95 \mathrm{GHz}$ designs appropriately to maintain $-20 \mathrm{~dB}$ return loss.

This termination is in close thermal contact with two TESs: an aluminum TES with a transition temperature $T_{c} \sim 1.2 \mathrm{~K}$ for lab tests and a $\sim 60 \mathrm{~m} \Omega$ titanium TES with a $T_{c} \sim 0.5 \mathrm{~K}$ for onsky observations. The saturation power (optical plus electrical power) needed to bring the TES temperature to $T_{c}$ is

$$
P_{\mathrm{sat}}=G_{c} T_{c} \frac{1-\left(T_{o} / T_{c}\right)^{\beta+1}}{\beta+1}
$$

where the conductance $G_{c}$ is evaluated at the transition temperature $T_{c}$ and the surrounding heat bath is $T_{o} \sim 280 \mathrm{mK}$.

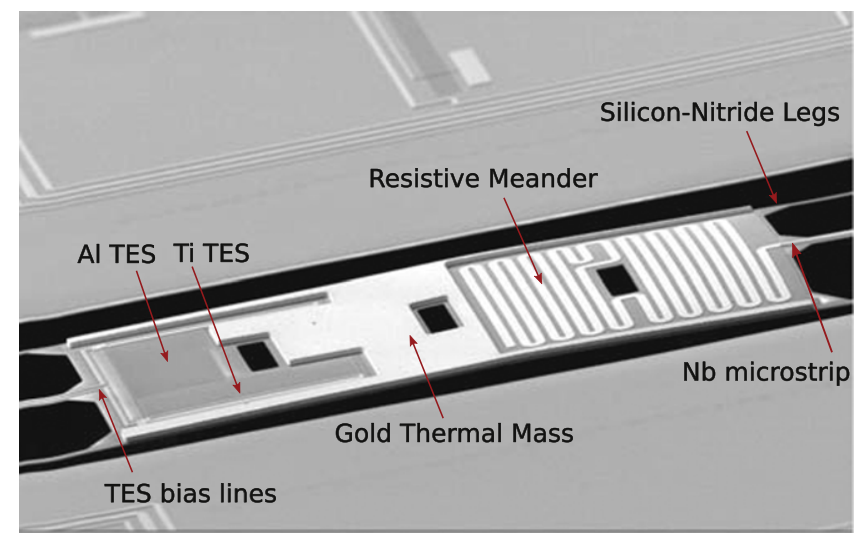

Figure 6. Electron micrograph of a released TES bolometer, illustrating its major components. The gold-meandered microstrip termination is at the right of the photograph and the TESs at left. The thicker gold film in the center of the island ensures thermal stability.

The exponent $\beta$ reflects the thermal carriers in the legs, where $\beta=1$ would correspond to electrons and $\beta=3$ would correspond to phonons in 3D materials. As discussed in Section 7.1, our typical devices are described by $\beta=2.1$. The aluminum $T_{c}$ affords the bolometer a high saturation power for use under a $300 \mathrm{~K}$ background, but has higher noise that would be unacceptable for astrophysical observations.

Each bolometer island is suspended from the tile by six isolation legs: one carrying the microstrip from the antenna, one carrying the TES DC lines, and four thinner legs for mechanical stability. Some newer devices use four legs, while SPIDER employs long meandered legs as described below. We tune the bolometer legs to achieve the design thermal conductance. We aim to keep the phonon noise subdominant to the photon noise, but also to keep the $G$ high enough that the detectors do not saturate under typical on-sky loading conditions, which for BICEP2 is 4-6 pW. We typically build in a safety factor of 2 beyond the expected maximum loading; for BICEP2 this resulted in $G_{c}=80-150 \mathrm{pW} \mathrm{K}^{-1}$, as described in Section 7. Two detector pairs in the corners have the microstrip opened between the antenna and bolometers such that the TESs do not receive optical stimulation through the microstrip feed. As described in Section 6.3, we use these four bolometers for diagnostic measurements and to monitor direct stimulation of our detectors by optical power that bypasses the microstrip circuitry and may thus not have the required spectral and polarization properties.

Thick evaporated gold (visible in Figure 6) is added to the bolometer islands to boost the heat-capacity by $C_{\mathrm{Au}} \simeq 0.5-0.3 \mathrm{pJ} \mathrm{K}^{-1}$, bringing the time constant $\tau=C / G$ to around a millisecond. We infer this from responses to a $1 \mathrm{~Hz}$ square-wave modulated broad-spectrum noise signal. The additional gold is important because of the strong electrothermal feedback of these devices; without it, our detectors would enter electrothermal oscillations throughout much of the TES transition.

We voltage bias the TESs into the transitions typically at half the normal resistance $R_{n}$ (i.e., $R_{\text {bias }} \simeq R_{n} / 2$ ), using $3 \mathrm{~m} \Omega$ parallel shunt resistors. We detect changes in the TES current using a SQUID-based time-domain multiplexing architecture, where all detectors in a set of 32 are sequentially read through a common set of lines (de Korte et al. 2003 and Stiehl et al. 2011). For BICEP2, our revisit rate of a given detector is 
$\sim 25 \mathrm{kHz}$, ensuring that we Nyquist sample the time-stream up to the $6 \mathrm{kHz}$ roll-off imposed by a $1.35 \mu \mathrm{H}$ series inductor.

Adapting this technology to the low photon loadings of SPIDER's long-duration balloon payload poses challenges in detector and instrument design. In order to take full advantage of the low photon noise levels available at $36 \mathrm{~km}$ altitudes, care must be taken to ensure that the bolometers achieve low noise levels and the surrounding instrument contributes minimal additional photon loading.

SPIDER employs several modifications to the BICEP2 detector design to reduce detector noise. A meandered leg design reduces conductance to $G \sim 15 \mathrm{pW} \mathrm{K}^{-1}$, reducing phonon noise while remaining within a similar footprint. This chosen $G$ is not limited by the anticipated loading of $\sim 4.5 \mathrm{~K}_{\mathrm{CMB}}$ but rather to have margin on the $300 \mathrm{~K}$ background in the laboratory while biased on the Al TES. Lower $G$ leads to naturally slower detectors, reducing the need to add heat capacity to the island with thick gold $(\mathrm{Au})$ to maintain stability. The long cabling in SPIDER prevents us from multiplexing faster than $\sim 20 \mathrm{kHz}$, so we control aliasing with larger inductors.

\section{FABRICATION}

We fabricate detectors in monolithic batches of 64 matched pairs at the Microdevices Laboratory at the Jet Propulsion Laboratory (JPL). We fabricate arrays on $350-800 \mu \mathrm{m}$ thick silicon substrates chosen for optimal optical coupling. We deposit $0.7-1.2 \mu \mathrm{m}$ low-stress silicon nitride films (LSN) and tune the internal stress to be as low as $150 \mathrm{MPa}$ to ensure mechanical integrity.

The TES normal state resistance $R_{n}$ and transition temperature $T_{c}$ are sensitive to thickness variability and chemical contamination, so we deposit and pattern our TESs before the millimeter wave circuitry on a flat and chemically clean surface. We first e-beam evaporate aluminum (Al) and pattern the $T_{c}=1.2 \mathrm{~K}$ TES. We DC-sputter titanium (Ti) and use inductively coupled plasma (ICP) etching to etch the $T_{c}=0.5 \mathrm{~K}$ TES for on-sky observing. Both films are immediately chemically passivated with RF-sputtered silicon dioxide $\left(\mathrm{SiO}_{2}\right)$ with ICP etched via holes to allow DC electrical contacts with subsequent layers. The RF bias ensures that the passivation patches have rounded edge walls so that subsequent films can make contact over steps. The top panel of Figure 7 illustrates these first steps. The $\mathrm{Al}$ and $\mathrm{Ti}$ films make a series $\mathrm{DC}$ connection and are later connected to $\mathrm{Nb}$ bias lines.

The millimeter wave circuits are fabricated from four films: a niobium ground plane, a $\mathrm{SiO}_{2}$ interlayer dielectric (ILD), an upper microstrip $\mathrm{Nb}$ conductor, and thin $\mathrm{Au}$ resistive termination. We DC-sputter the $\mathrm{Nb}$ films and pattern the ground plane with lift-off, a technique where we first deposit photoresist and then dissolve it from under the metal film to be removed. In principle, this film could be etched, but we suspect that the Ti $T_{c}$ can be altered by this step despite the intervening protect layer. The $\mathrm{Nb}$ ground film defines the antenna slots, bandpass filter inductors, holes for bolometer release, and safety holes under the bond-pads. This film also fills the bias vias down to the TESs. Lift-off provides rounded side-walls and thus ensures step-coverage of subsequent films. The $\mathrm{Nb}$ ground-plane covers more than $90 \%$ of the $100 \mathrm{~mm}$ wafer, serving as both the DC and RF ground for the detector array.

We have explored several ILD materials, although all currently deployed devices use RF-sputtered silicon dioxide. Thickness uniformity of this film is crucial for detector
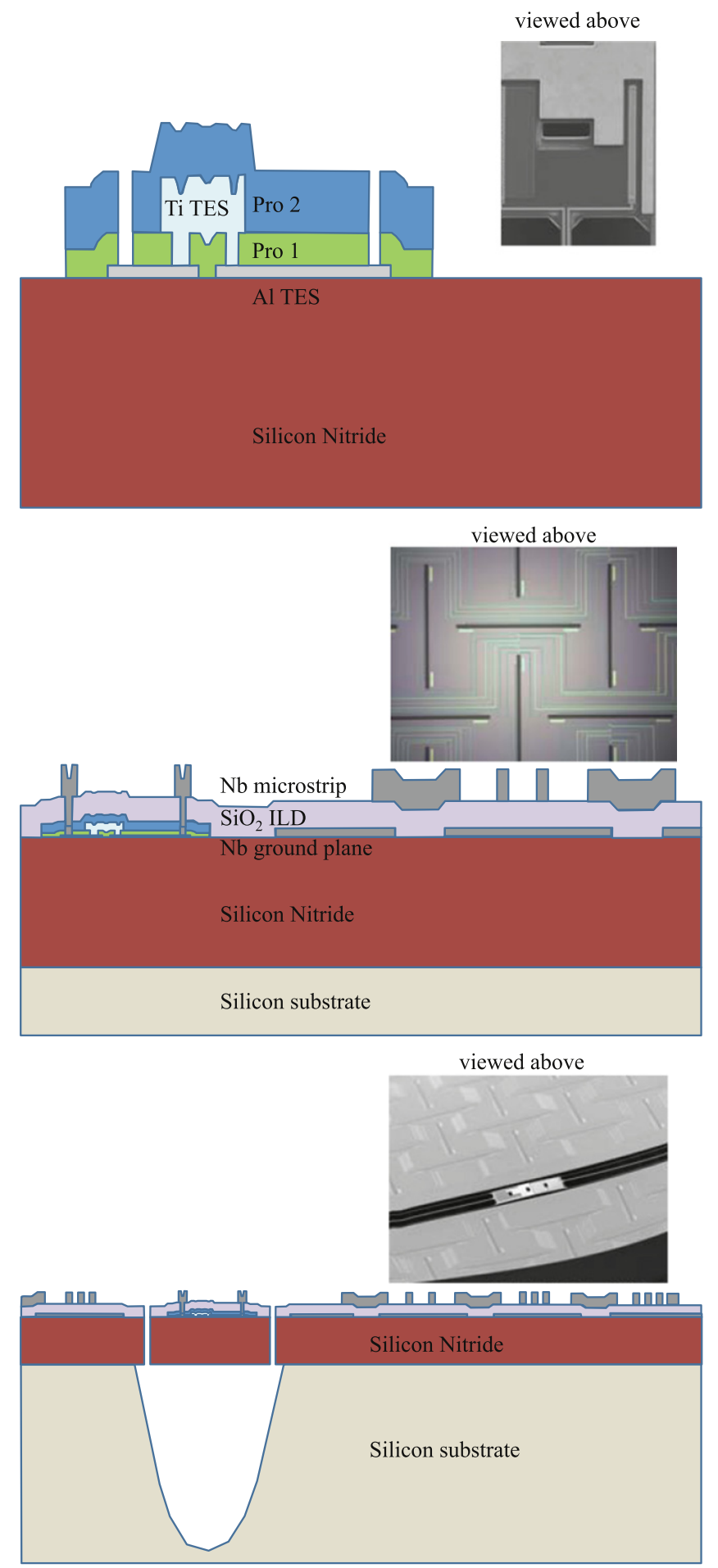

Figure 7. Cross-section of films in order of fabrication The aspect ratio between radial and normal dimensions are distorted for clarity. We include photos of how the device looks face-on for reference. Upper: deposition and etching of films for the TESs and their protect layers. Middle: deposition and etching of antenna and microstrip features. Lower: release of TES bolometer.

uniformity across the array. A 6 inch $\mathrm{SiO}_{2} \mathrm{RF}$ sputtering target and substrate rotation help achieve thickness uniformity to better than $7 \%$ across the array. We use an ICP reactive ion etch system to etch release holes around the bolometers and vias that allow for a DC connection to the buried TES structures in the subsequent $\mathrm{Nb}$ metallization fabrication step. 
Table 1

Summary of Processing Steps

\begin{tabular}{lll}
\hline \hline Material/Function & Deposition & Etchant \\
\hline 1. Low-stress nitride & High temp LPCVD & $\mathrm{CHF}_{3} \mathrm{ICP}-\mathrm{RIE}$ \\
2. Aluminum TES & e-beam evaporation & Liftoff \\
3. $\mathrm{SiO}_{2}$ Pro-1 & RF sputtered & $\mathrm{ICP}, \mathrm{CHF}_{3} / \mathrm{O}_{2}$ etch \\
4. $\mathrm{Titanium} \mathrm{TES}_{\text {5. } \mathrm{SiO}_{2} \text { Pro-2 }}$ & sputtered & $\mathrm{ICP}$, Freon- 1202 \\
6. $\mathrm{Niobium} \mathrm{Ground} \mathrm{Plane}_{\text {7. } \mathrm{SiO}}$ ILD & RF sputtered & ICP, $\mathrm{CHF}_{3} / \mathrm{O}_{2}$ etch \\
8. Gold resistor & RF sputtered & Liftoff \\
9. Niobium Microstrip & e-beam evaporated & ICP CHF $/ \mathrm{O}_{2}$ etch \\
10. Gold heat capacity & sputtered & ICP, Freon-1202 \\
11. Silicon release & e-beam evaporated & Liftoff \\
& substrate & STS DRIE $/ \mathrm{XeF}_{2}$ \\
\hline
\end{tabular}

Note. Acronyms defined in text.

We form the resistive terminations on the bolometer islands with e-beam deposition and lift-off patterning. Finally, we DCsputter the upper $\mathrm{Nb}$ microstrip conductor and etch from that film the microstrip feed network, band-defining filter capacitors, and DC bolometer bias lines and bond-pads using a freon etch. We have found that lift-off techniques (used for BICEP2) and chlorine-based $\left(\mathrm{BCl}_{3}\right)$ etches can contaminate the $\mathrm{Nb}$ films at this step, resulting in unacceptable millimeter wave losses in our circuitry as discussed in Sections 5 and 6.2. The middle panel of Figure 7 illustrates these intermediate microwave steps that define ground plane, ILD, and microstrip traces.

The final fabrication steps release the bolometer islands, illustrated in The bottom panel of Figure 7 . We etch through the $\mathrm{LSN}$ with $\mathrm{CHF}_{3}$ in an inductively coupled plasma reactive ion etch ICP-RIE system, exposing bare silicon under the release holes. We e-beam evaporate and lift-off thick gold onto the islands to add heat capacity and control the required readout bandwidth. We use an STS deep reactive ion system to etch from the front side and remove silicon from the release holes. This Bosch etching process uses a combination of etching and passivation steps to cut vertically through the silicon wafer, after which we undercut the bolometers with a $\mathrm{XeF}_{2}$ release (Turner et al. 2001). $\mathrm{XeF}_{2}$ attacks the remaining exposed silicon isotropically, so the small hole pattern in the photoresist is pre-determined to release only the islands and legs and to not undercut the remaining antenna structures. Finally, we use the STS etcher to cut out the square tile with holes for alignment pins. Table 1 summarizes these fabrication steps.

\section{MATERIAL PROPERTIES FOR THE MILLIMETER WAVE CIRCUITS}

We separately characterize the material properties used in the design of the antenna feed and filter, in particular the $\mathrm{Nb}$ penetration depth and the ILD dielectric constants. These material parameters are poorly characterized in the literature for millimeter waves, and they can depend on details of the processing. We also need to characterize loss properties and monitor stability over time. To address these concerns, we have fabricated companion tiles of test devices where each detector pair receives power through a single polarization broadband integrated antenna. The microstrip feed evenly divides power in a microstrip tee-junction between a device under test (DUT) and a reference bolometer to divide away optical effects from the antenna and fore optics. We measure the spectral response
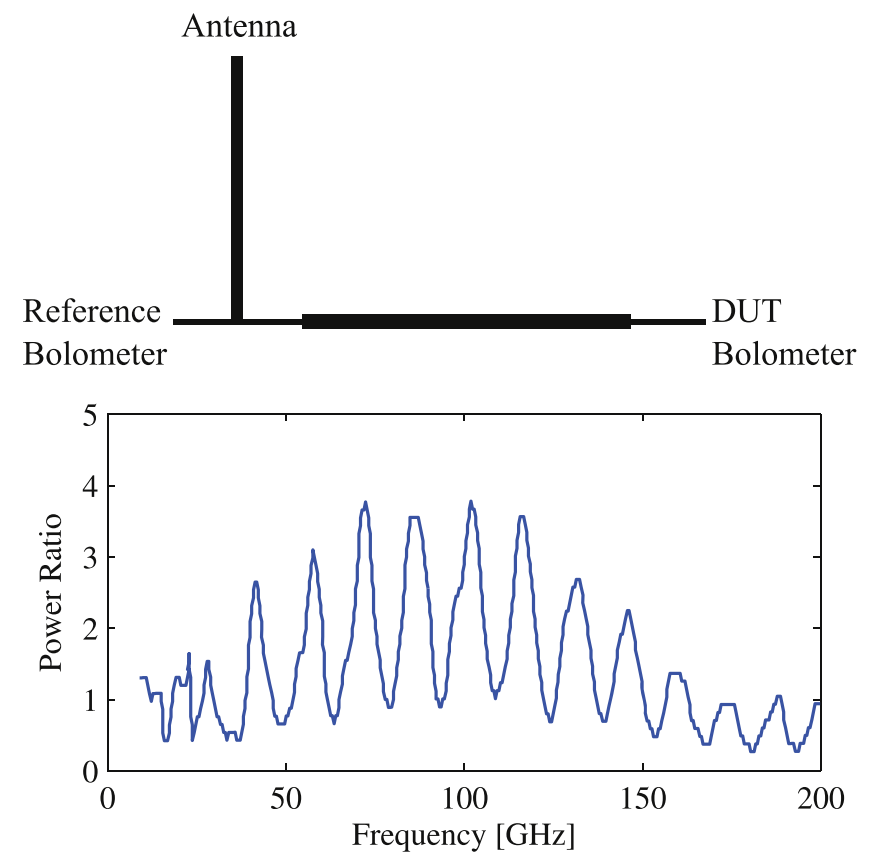

Figure 8. Upper: schematic of a test device for microstrip wave speed, where the mismatched microstrip segment at right forms a Fabry-Perot cavity. Lower: standing wave pattern in a device's FTS spectrum. The period is proportional to the transmission line wavespeed.

of the bolometers through Fourier transform spectroscopy (FTS) with a Martin-Puplett interferometer (Martin \& Puplett 1970), combining the detector time-streams and encoder readings from the moveable mirror's translation stage to form interferograms. We low-pass filter, zero-path difference, and Hanning apodize the interferogram before Fourier transforming the interferogram into the frequency response $S(\nu)$.

We use one device for microstrip wave speed measurements. The DUT is a microstrip Fabry-Pérot cavity with an impedance intentionally mismatched from the surrounding lines. The standing wave pattern in the spectrum of the bolometer behind the cavity can be used to determine wave speed. (See Figure 8 for a schematic and data sample.) The wave speed is a function of both the ILD dielectric constant $\epsilon_{r}$ and the $\mathrm{Nb}$ penetration depth $\Lambda$. Alternatively, the band-defining filter resonance is determined by series inductance dominated by the CPW magnetic inductance. Data from the band locations and test devices allow us to solve for both $\epsilon_{r}$ and $\Lambda$ and we summarize these numbers is Table 2 for different materials.

Another test device measures loss per length in our transmission lines, where the DUT is a stretch of line that is several wavelengths long. We determine loss as a function of frequency by forming the ratio of FTS spectra seen through the long line and the reference bolometer. While the loss tangent has been shown to be dominated by loss in dielectrics, and can be quite low in some dielectric systems, our measurements determine loss in the microstrip as fabricated. Thus far we have tested PECVD $\mathrm{SiO}_{2}$, PECVD $\mathrm{Si}_{x} N_{y}$, and evaporated $\mathrm{SiO}_{2}$ in this manner.

This loss test capability became particularly important during a period of time when the optical efficiencies of our fabricated devices were unusually poor. This test program allowed us to show that the effective loss tangent of our microstrip had a $\nu^{2}$ frequency dependence (see Figure 9) when using all three of the dielectrics above. This common problem present with three 
Table 2

Material Properties at Millimeter Waves

\begin{tabular}{lc}
\hline \hline Parameter & Value \\
\hline $\mathrm{SiO}_{2} \epsilon_{r}$ & 3.9 \\
$\mathrm{Si}_{x} N_{y} \epsilon_{r}$ & 7.0 \\
$\mathrm{Nb} \Lambda$ & $0.1 \mu \mathrm{m}$ \\
\hline
\end{tabular}

ILDs from different deposition systems suggested that the loss was generated in the $\mathrm{Nb}$ films. Ultimately, these measurements helped us to identify and correct a modest tensile stress in the $\mathrm{Nb}$ as deposited by sputtering. Further, the loss appears to be sensitive to the reactive ion etch chemistry used in the $\mathrm{Nb}$ process. Controlling the film stress at a nominal compressive -100 to $-300 \mathrm{MPa}$ and changing the $\mathrm{Nb}$ etchant from $\mathrm{BCl}_{3}+\mathrm{O}_{2}$ to $\mathrm{Fl}_{2}+\mathrm{O}_{2}$ resulted in lower loss tangent with a $\nu^{1}$ dependence (see Figure 9).

\section{BEAM SYNTHESIS}

The antenna array combines waves from the sub-antennas with equal amplitude and phase to synthesize a uniform illumination, which generates a sinc pattern in the antenna far field. Using a test cryostat, we characterized the antenna far field pattern and confirmed that the antenna has a 1/e beam waist of $4.1\left(\right.$ FWHM $\left.\sim 14^{\circ}\right)$ and side-lobe level of $-12 \mathrm{~dB}$ relative to peak response, as expected from the design. A sample far-field pattern is shown in Figure 10, taken in a cryostat without lenses or a stop. For a detailed beams characterization in the complete BICEP2 and Keck Array cameras, we refer the reader to the BicEP2 and Keck Array Beams Paper (Bicep2 and Keck Array Collaborations IV 2015).

Our ground-based experiments difference detector pairs at the time stream level to suppress unpolarized common-mode noise from the atmosphere. However, differencing at this early point in the analysis pipeline can allow temperature anisotropies to leak into polarization if the detector beam patterns are mismatched. This is an especially acute challenge to small aperture experiments because the beam size couples to large temperature gradients at degree scales.

Our team has developed an analysis technique, called deprojection, that discards contaminated modes. As described in the BICEP2 Systematics Paper (Bicep2 Collaboration III 2015), the pipeline can remove temperature leakage through relative-gain mismatch (monopole moment of beams), first derivatives of temperature through displaced centroids (dipole moment of beams), and second derivatives of temperature through differential beam widths and ellipticities (quadrupolar moments of beams).

The detector design is most prone to centroid displacement, as characterized in the Beams Paper (Bicep2 and Keck Array Collaborations IV 2015). We have identified two mechanisms responsible for beam displacement and implemented design and fabrication fixes to suppress their contamination below the $r=0.1$ level (O'Brient et al. 2012). These fixed detectors are deployed in Keck Array and SPIDER and Figure 12 shows the centroid steer before and after these corrective measures.
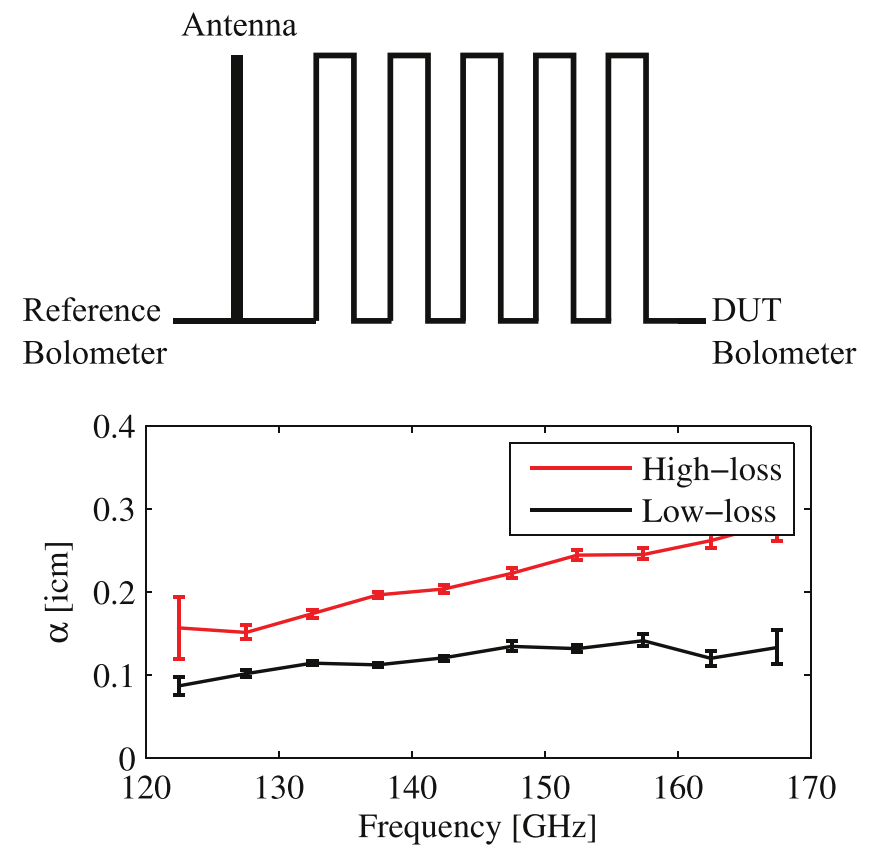

Figure 9. Upper: schematic of a test device for microstrip loss rates, where the DUT is a stretch of transmission line several wavelengths long. Lower: sample spectra showing power loss per unit length $(\alpha=-d(\log P) / d x)$ in films exhibiting low and high loss. Nonlinear increase in loss with frequency has been an indicator of bad process parameters in need of adjustment.

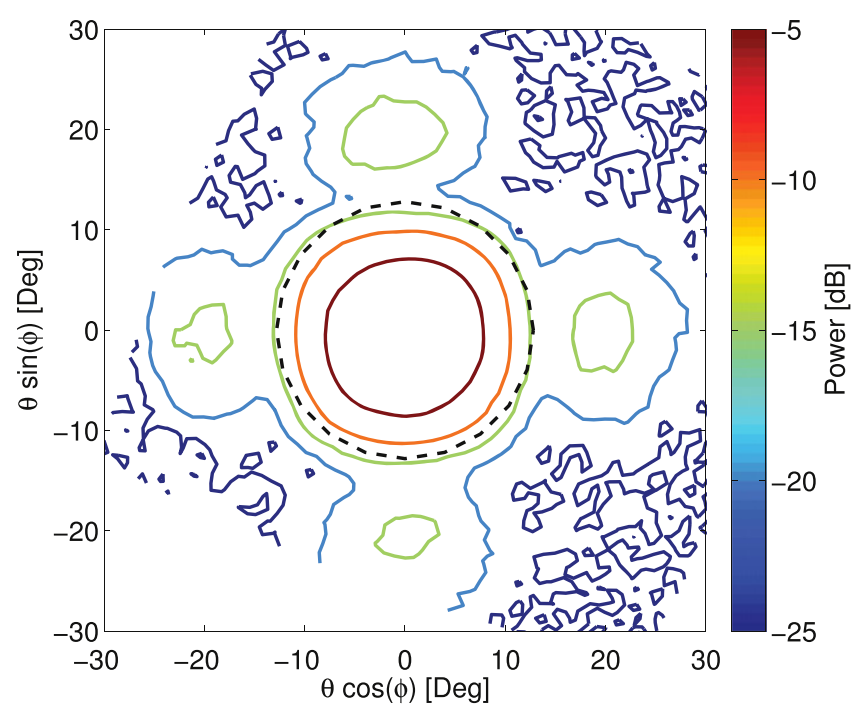

Figure 10. Sample far-field detector pattern measured in a test cryostat without an optical stop. Power is normalized to peak on boresight and the dashed line indicates where the $f / 2.2$ camera stop would lie.

\subsection{Parasitic Microstrip Cross-talk}

The microstrip lines in the feed networks' horizontal arms must fit between the slot sub-radiators, crossing them only at the intended feed points. To achieve this, the lines must be in close proximity, and for the $150 \mathrm{GHz}$ BICEP2 devices, many lines are separated by only $\sim 10$ dielectric thicknesses. This separation would be adequate to avoid cross-talk for short stretches of lines, but some of the line pairs run parallel for over four wavelengths. The longest of these pairs are those running from the sides to the center to begin the horizontal tree arms, coupling power to one of the two lines they intentionally split 

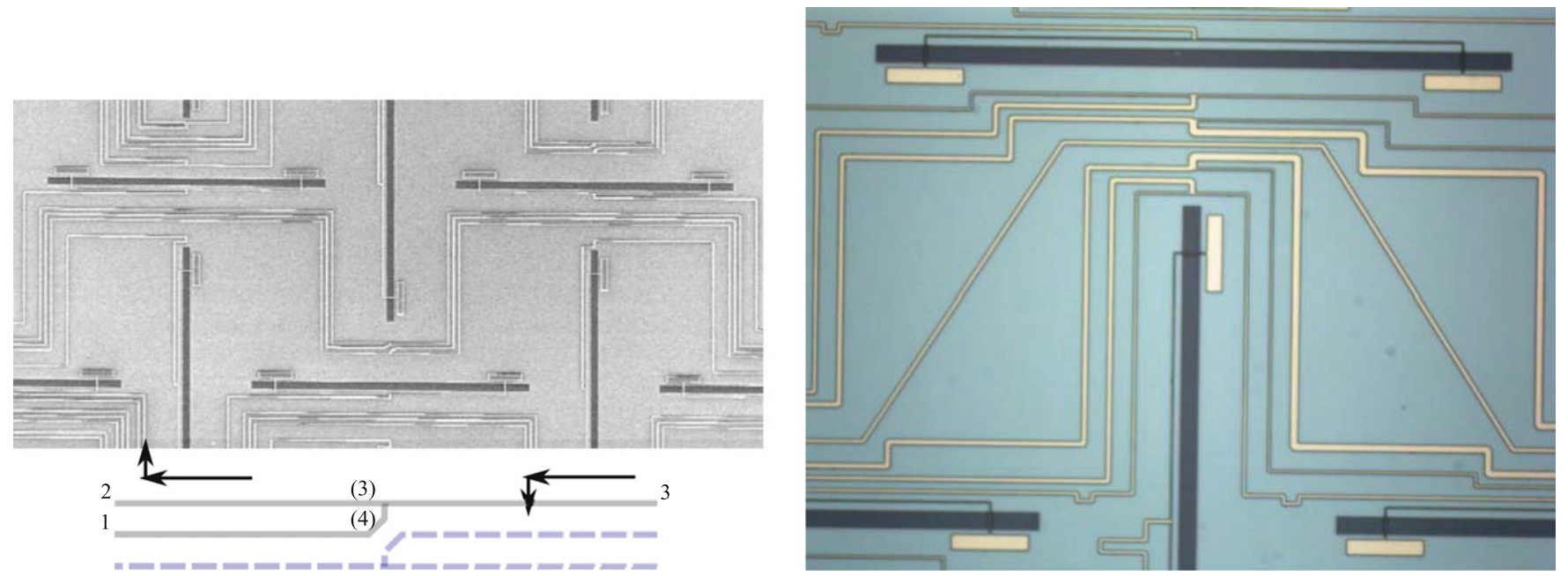

Figure 11. Details of microstrip layout in the center of the antenna feed. Left: center of the BICEP2-era design. The Cartoon under the picture shows the effective coupler circuit and the ports corresponding to the scattering parameters in Equation (9). The arrows are phasors that illustrate how the intended waves (horizontal) combine with the parasitic coupled waves (vertical). Right: modern design with reduced coupling due to greater spaced lines. The extra lengths of line that form compensating phase lags are visible (e.g., bottom of picture), although these are not necessary with the suppressed microstrip coupling.
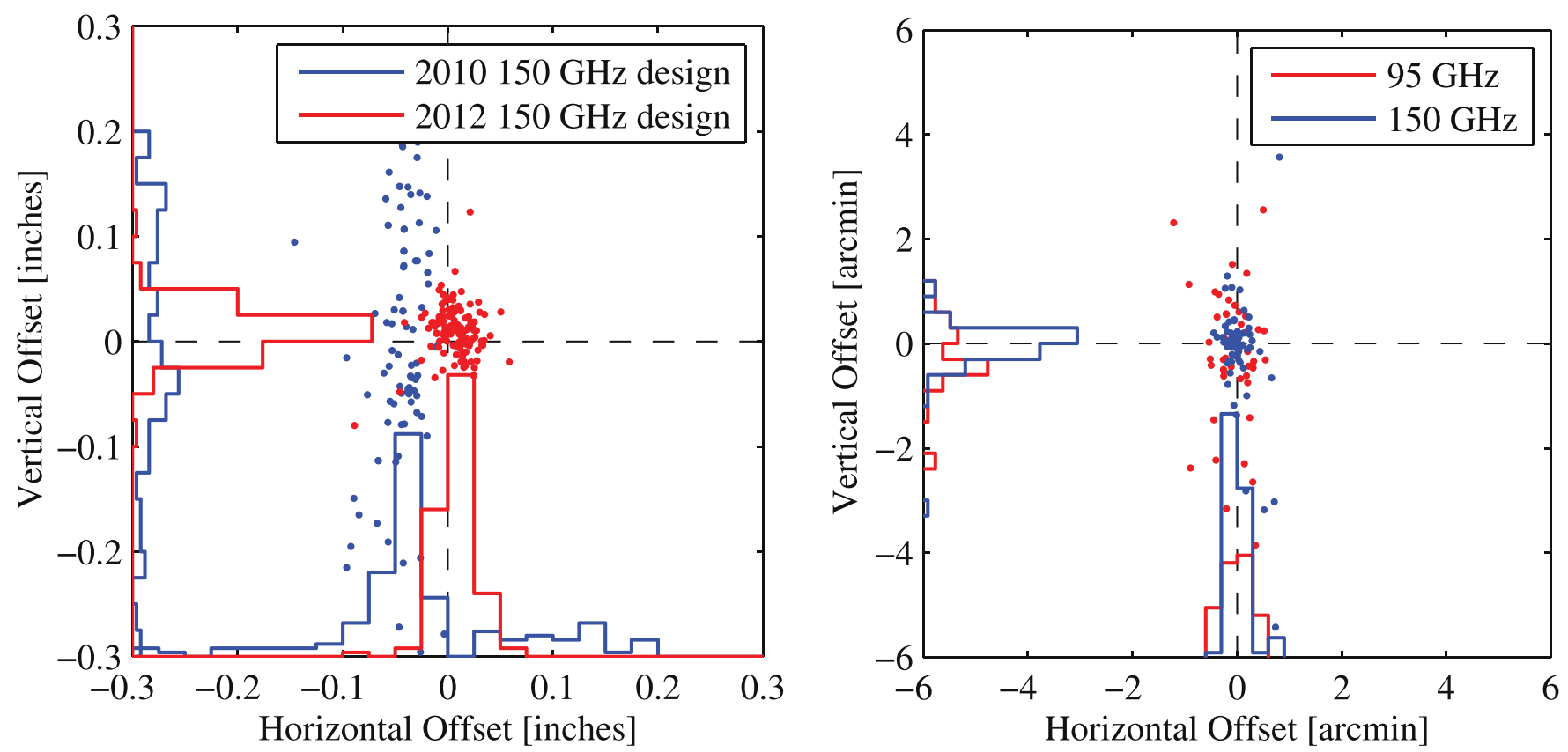

Figure 12. Left: beam centroid displacements in the near field before and after fixes were made to the etch recipe. The performance shown in left panel would allow a BICEP1-style pipeline without deprojection to constrain $r$ to 0.1 (Takahashi et al. 2010). The deprojection pipeline described in (Bicep2 and Keck Array Collaborations III 2015) allows $r$ to be constrained to yet lower levels. Right: beam centroid displacements in the far field for two different colors after the recipe was fixed.

power between, as seen in Figure 4 and summarized in the bottom of the left panel of Figure 11.

In a reverse-time picture, power from port 1 (defined in Figure 11) should be evenly divided between ports 2 and 3. The currents in a pair of parallel lines can be expressed as a superposition of even and odd modes, where the even mode has the same current magnitude and directions in each line and the odd mode has the same magnitude but opposite directions. Coupling between the two parallel lines, however, results in the two modes having different field concentrations in the air and dielectric volumes, and so induces a difference in wave speeds between the modes. Waves passing from port 1 to internal port (4) thus also couple waves into internal port (3) that lag by $90^{\circ}$ in phase:

$$
\begin{aligned}
S_{(4) 1} & =\left(S_{(4) 1}^{e}+S_{(4) 1}^{o}\right) / 2=\left(e^{-j k_{e} \ell}+e^{-j k_{o} \ell}\right) / 2 \\
& =e^{-j\left(k_{e}+k_{o}\right) \ell / 2} \cos \left(\left(k_{e}-k_{o}\right) \ell / 2\right) \\
S_{(3) 1} & =\left(S_{(3) 1}^{e}+S_{(3) 1}^{o}\right) / 2=\left(e^{-j k_{e} \ell}-e^{-j k_{o} \ell}\right) / 2 \\
& =-j e^{-j\left(k_{e}+k_{o}\right) \ell / 2} \sin \left(\left(k_{e}-k_{o}\right) \ell / 2\right)
\end{aligned}
$$

where $k_{e}$ and $k_{o}$ are the even and odd mode wavenumbers and $\ell$ is the parallel coupling length. This circuit acts as an unintentional reverse-wave coupler, where impedance mismatches between even and odd modes are small, but 
wavespeeds are not (Hammerstad \& Jensen 1980). The waves entering the microstrip tee-junction from internal port (4) add in quadrature to the intended waves from internal port (3), advancing the total phase on the side opposite the vertical tree and retarding it on the other. This phase-step steers the beams off boresight away from the vertical summing trees and is where the left-right symmetry splitting occurs in the feed. This effect produces the repeatable horizontal centroid steer seen in the cameras' near field. We have reproduced this in simulations using HFSS that account for the upper conductor finite thickness.

As seen in the right panel of Figure 11, we have re-designed the antenna feeds for the Keck Array and SPIDER detectors to greatly increase spacing between the lines and therefore reduce the parasitic coupling effect. We also include adjustable phaselag lines before each slot to remove residual phase-error and to synthesize matched beams, although the current antenna-feeds' increased spacing renders the corrective phases unnecessary. These phase-lags are visible in the bottom of the right picture in Figure 11.

\subsection{Niobium Contamination}

Magnetic fields can penetrate into a superconductor by a characteristic depth $\lambda_{\text {eff }}$. Impurities in niobium films scatter Cooper pairs with a mean free path $\ell$, increasing the penetration depth beyond the London depth $\lambda_{L} \sim 50 \mathrm{~nm}$ of pure niobium to $\lambda_{\text {eff }}=\lambda_{L} \sqrt{\xi_{o} / \ell}$, where $\xi_{o} \sim 40 \mathrm{~nm}$ is the Cooper pairs coherence length (Tinkham 1996). As displayed in Table 2, our films are typically measured to have $\lambda_{\text {eff }} \sim 100 \mathrm{~nm}$, suggesting mean-free path of $\ell=10 \mathrm{~nm}$, well within the "dirty" limit where film cleanliness can impact circuit performance.

Nonuniform contamination can produce nonuniform kinetic inductance, which can spatially perturb the wavespeeds in the microstrip summing tree. Variations in wavespeed can steer beams off boresight. Additionally, the summing tree does not treat the two polarizations identically, and as a result, they can be steered differentially. We have observed that the tiles with the largest scatter in beam centroid position correspond to those with the largest vertical dipole components. We also expect the tree to induce larger steering in the vertical than horizontal because slots along rows combine immediately in the horizontal tree, resulting in less integrated phase error than those along columns that combine in the vertical tree after horizontal summing.

We have built models for how our antenna-feeds perform with film gradients. We subdivide the circuit into short sections of transmission lines and tee-junctions and use each section's location in the detector to assign unique film properties. We construct simple scattering matrices for each section and cascade them into one large $289 \times 289$ matrix per polarization (Bodharamik et al. 1971). From this we can compute slot illumination patterns and thus far-field patterns. We find that $20 \%$ variations in $\lambda_{\text {eft }}$ across the array can produce differential pointing that is $10 \%$ of the beam FWHM in the vertical directions, and half that on the horizontal, similarly matching our observed scattering in pointing.

We defined our microstrip lines in early tiles with the lift-off technique that we use for the $\mathrm{Nb}$ ground plane, as described in Section 4. Several devices have shown discoloration in this step, leading us to speculate that the $\mathrm{Nb}$ leaches organic materials from the resist during lift-off. These observations and modeling inspired a switch to an etch-based means of defining the $\mathrm{Nb}$ microstrip lines (described in Section 4). This simple fix reduced the scatter in centroid location to $\sim 1 \%$ of Gaussian width $\sigma$, and the right panel of Figure 12 shows the centroid alignment between polarization pairs for both colors of deployed focal planes.

\subsection{Direct Stimulation of Bolometers}

Optical power is meant to reach the bolometers only through the antenna and microstrip feed network. However, photons can directly excite responses in the detectors which is of particular concern in a design where there are no horn blocks to shield the sensors themselves. In BICEP2 pre-deployment testing of early generations of detectors, response to out-of-band power was detected at levels $3 \%-4 \%$ of the total response, with near field and far field angular response patterns consistent with direct stimulation of the bolometer islands. Simulations in HFSS and CST suggest that the ground plane on the bolometer islands can be inductively held at a different voltage than the surrounding ground plane through the microstrip ground that we deposited only on one leg in early generation prototypes. This voltage can drive millimeter-wave currents through the gold-termination, resulting in a direct stimulation of the detectors not through intended antenna and microstrip feed. Steps taken prior to BICEP2 deployment to minimize this coupling included the addition of metal mesh low-pass edge filter above the focal plane and several design changes to the bolometer islands themselves. The island leg design was modified to narrow the width of the opening in the ground plane surrounding the island, and ground plane continuity was extended onto the island by metalization of the four outer support legs.

With these modifications in place, we have taken BICEP2 and Keck Array $150 \mathrm{GHz}$ camera optical efficiency measurements with and without high-pass "thick-grill" filters that obstruct power below $200 \mathrm{GHz}$; after accounting for the filter's filling factor, we found that the optical response through the filter was $\sim 0.5 \%$ of that without filtering. This small leakage suggests limited "blue-leaking" that would allow above-band power to excite response in the bolometer. We have also measured the response of our detectors to a chopped thermal source on boresight through a polarizing grid at different angles; we have found that the crossed response is similarly $\sim 0.5 \%$ of the co-polarized response, consistent with known multiplexer cross-talk levels. If the detectors are acting as a direct absorber, then their small area compared to the antenna's should provide a broad angular response. As a result, we expect that the $f / 2.2$ stop in the cameras for these experiments helps limit the direct stimulation and we have found that tests of devices in cameras with faster optics can have higher direct stimulation levels.

\section{ARRAY PROPERTIES AND UNIFORMITY}

Our high attained sensitivities are a result of our high detector yield, a figure that depends on attaining uniform detector properties across the array. This uniformity also helps to mitigate some potential sources of systematic error. This section describes array uniformity of a variety of properties. 


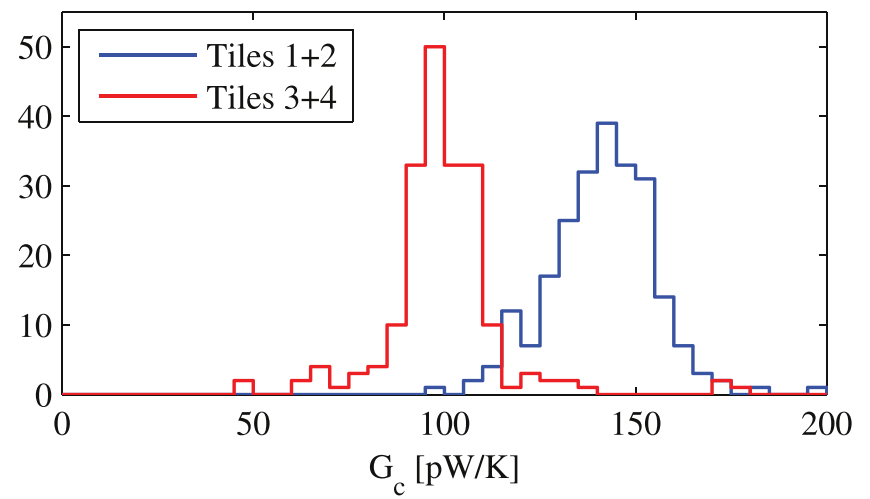

Figure 13. Thermal conductance of BICEP2 prototype tiles at $T_{c}$. This figure demonstrates repeatability within and between tiles.

\subsection{Bolometer Thermal Conductance $G(T)$}

Thermal fluctuation noise across the bolometer legs is the largest internal source of noise in BICEP2, and is given by

$$
\mathrm{NEP}_{G}^{2}=4 k T_{c}^{2} G_{c} F\left(T_{c}, T_{o}\right)
$$

where the "Mather factor" $F\left(T_{c}, T_{o}\right)$ (Mather 1982) accounts for thermal gradients across the bolometer legs and is roughly 0.5 for our detectors. The saturation power of the detector is given in Equation (8). Note that the leg thermal conductance $G$ is a function of temperature.

The legs' total parallel thermal conductance $G$ needs to be chosen to keep thermal carrier noise subdominant to photon shot and Bose noise, but avoid saturation under on-sky loading. We try to have the total saturation power (Joule heating from the bias circuit plus optical loading on the detectors) exceed the optical loading ( 4-6 pW at $150 \mathrm{GHz}$ in BICEP2) by a factor of two. This aggressive safety factor is only possible if we have tight control of our processing parameters that determine $G$ and $T_{c}$ (discussed in the next subsection). Our achieved repeatability between detectors in a tile and from tile-to-tile are shown in Figure 13. Our greatest variations in this parameter happen between fabrication runs, and the histograms are grouped by pairs of tiles fabricated at common times to illustrate how this parameter varies between those runs. The histograms' widths indicates the variability within a fabrication run. Our timedivision multiplexing readout requires that detectors in a common readout column be commonly voltage biased (Battistelli et al. 2008 and Stiehl et al. 2011), and our control of $G$ within a tile allows this.

The $\mathrm{XeF}_{2}$ release only has a 10:1 selection ratio between silicon nitride and silicon, and variation in bolometer leg thicknesses from nitride etching would seriously compromise these numbers. For this reason, we oxidize the wafers prior to nitride deposition. The thin $\sim 10 \mathrm{~nm} \mathrm{SiO}_{2}$ film has a much higher 100:1 selection ratio between oxide and silicon and thus protects the legs from $\mathrm{XeF}_{2}$ attack (Williams et al. 2003). This oxide film ensures more uniform leg cross-sections across the detector arrays, and thus more uniform bolometer $G$ s.

The thermal conductance of the bolometer legs varies with temperature as $G \sim T^{\beta}$. Figure 14 shows best fit exponents for a typical tile, extracted from $I V$ curves at several bath temperatures between 0.28 and $0.8 \mathrm{~K}$ in a test cryostat where the sensors are only exposed to internal $4 \mathrm{~K}$ thermal radiation. These particular detectors are well described by $\beta=2.1$, and all tiles have measured $\beta$ between 1.9 and 2.3.

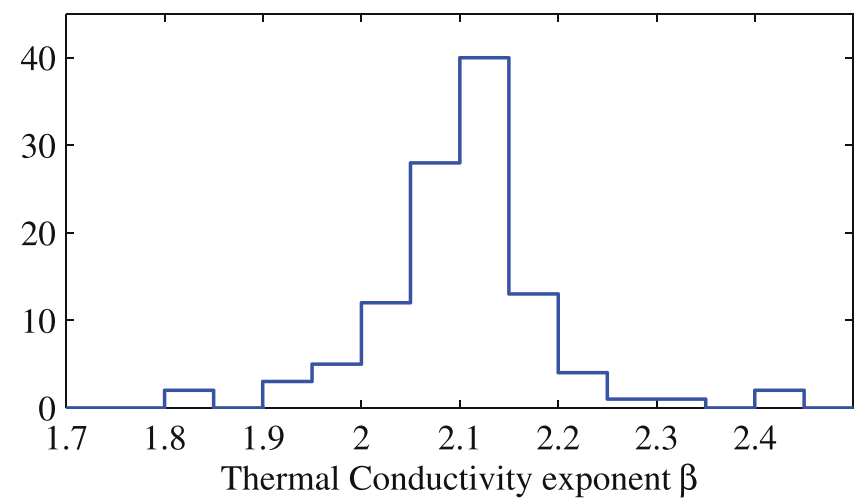

Figure 14. Thermal conductance exponent $\beta$ for a single SPIDER tile.

In the absence of scattering, both heat capacity and thermal conductance scale as $T^{d}$, where $d$ is the number of dimensions greater than the dominant thermal phonon wavelength

$$
\lambda_{\text {phonon }}(T)=\frac{h c_{s}}{k T} .
$$

Given a sound speed of $c_{s} \sim 6500 \mathrm{~m} \mathrm{~s}^{-1}$ (Holmes et al. 1998) in bulk silicon nitride, we expect the thermal phonon wavelength in the legs to vary from $0.6 \mu \mathrm{m}$ at the hot ends to $1.1 \mu \mathrm{m}$ at the cold ends. With legs that are 4-10 $\mu \mathrm{m}$ wide and 600-1000 $\mu \mathrm{m}$ long, but only $\sim 1.5 \mu \mathrm{m}$ thick, we expect to see thermal behavior similar to a two-dimensional phonon gas with $\beta \sim 2$. If the lateral dimensions were both smaller than $\lambda_{\text {phonon }}$ and all were smaller than the mean free path under scattering $\lambda_{\mathrm{mfp}}$, then the "one-dimensional" conduction through $N$ legs will conduct with $G=4 \mathrm{Ng}_{Q} T$, where four "massless" modes without a lowfrequency cut-off participate at low temperatures and where $g_{Q}=\pi^{2} k_{b}^{2} / 3 h=0.95 \mathrm{pW} \mathrm{K}^{-2}$ is the quantum conduction of each mode (Rego \& Kirczenow 1998; Schwab et al. 2000). This predicts a maximum conduction of $12 \mathrm{pW} \mathrm{K}^{-1}$ at $0.52 \mathrm{~K}$ for BiCEP2, clearly disfavored by the data in Figure 13. Twodimensional conduction through $N$ legs of width $w>\lambda_{\text {phonon }}$ only has three massless modes, so $G=3 N \sigma_{2 \mathrm{D}} w T^{2}$, where $\sigma_{2 \mathrm{D}}=7.2 \mathrm{pW} / \mu \mathrm{m} \mathrm{K}^{3}$ (Holmes 1998). The maximum possible $G_{c} \sim 230 \mathrm{pW} \mathrm{K}^{-1}$ for BICEP 2 can accommodate the measured $G$ values.

Phonon scattering can decrease conductivity by $\lambda_{\mathrm{mfp}} / L$, where $L$ is the leg length. In the case of scattering from surface imperfections with an rms $\eta$, this mean free path is $(1+f(T)) /(1-f(T)) \mathrm{D}$ (Ziman 1963), where $D$ is the leg's shortest thermally active dimension and the specularity $f(T)$ is

$$
f(T)=\exp \left[-16 \pi^{2}\left(\frac{\eta}{\lambda_{\text {phonon }}(T)}\right)^{2}\right] .
$$

SEM images of our bolometers (such as Figure 6) suggest a top surface smooth to $\eta<10 \mathrm{~nm}$. We see little optical evidence of the $\mathrm{XeF}_{2}$ attacking the silicon nitride, which suggests that the back sides of the legs are also smoother than the thickness of the $10 \mathrm{~nm}$ oxide layer. These variations suggest a mean-free path $\lambda_{\mathrm{mfp}} \gtrsim 200 \mu \mathrm{m}$, which provides the expected inverse dependence of $G$ on $L$ and predicts a reduction of $G$ of order unity from maximum $2 \mathrm{D}$ conduction, as seen in our measurements (as seen in Figure 13). 
The specularity depends on temperature $T$ through the phonon wavelength $\lambda_{\text {phonon }}(T)$, which means that this efficiency factor can alter the thermal conduction exponent. The variations $\eta$ are small compared to the thermal phonon wavelength but still modifies the expected conductivity to an effective power law of $\beta=2.5$. The rms variations are likely smaller than this bound, so we can only say that we expect $\beta$ to be between 2.0 and 2.5, consistent with our measurements. More importantly, the repeatable and uniform $\beta$ and $G$, both within and between tiles, achieved through the oxidation of the silicon substrate to control surface smoothness and leg thickness, allows for repeatable saturation powers of our detectors.

\subsection{Transition Temperature $T_{c}$}

The balance between the between the internal ${ }^{3} \mathrm{He} /{ }^{3} \mathrm{He} /{ }^{4} \mathrm{He}$ fridge cooling power and thermal loading lets the focal plane cool to $\sim 250 \mathrm{mK}$. For BICEP2 and Keck Array, we use heaters to operate the focal plane (bolometer thermal bath temperature) at an elevated $280 \mathrm{mK}$ so we have margin to control this temperature through active feedback loops while still maintaining photon-noise-limited sensitivity. These temperature controllers provided a safeguard against focal-plane drifts and our systematics paper demonstrates that with these controllers, such drifts added negligibly to the B-mode power spectrum (Bicep2 Collaboration III 2015). SPIDER eschewed temperature control and achieved $\sim 10 \mathrm{mHz}$ stability from their sorption fridges alone (Gudmundsson et al. 2015). With this cryogenic system and bolometer legs with $\beta=2.1, \mathrm{NEP}_{G}$ is minimized at an island temperature of $515 \mathrm{mK}$, although this is a broad minimum with a gentle slope at higher temperatures. For on-sky operation, electrothermal feedback locks the bolometer to the titanium TES's $T_{c} \sim 520 \mathrm{mK}$. By selecting a pure superconductor for this TES instead of a bilayer (Gildemeister et al. 1999; Myers et al. 2005 and Fabrega et al. 2009), we obtain repeatable transitions devoid of the multiple transitions that can result from a bilayer's complicated chemistry. Deposition on bare wafers and passivation of oxide protection layers also help maintain uniform values between and within tiles. As seen in Figure 15, our titanium $T_{c}$ is uniform across tiles and even between tiles, maintaining uniform saturation powers (Equation (8)) within a multiplexer column, necessary to find a common bias point.

\subsection{Normal Resistance $R_{n}$}

TES bolometers will only experience electrothermal feedback if voltage biased, and we bias our nominally $\sim 60 \mathrm{~m} \Omega \mathrm{Ti}$ TESs with a $3 \mathrm{~m} \Omega$ shunt resistor. The Al TES is even higher in resistance, so both are far larger than the shunt. However, this condition is only met if the Ti TES resistance is repeatably this large. We also need them repeatable in value so the detectors will have a common latch resistance and thus a common bias point where they will experience strong feedback. Figure 16 shows that these values are indeed uniform between and within tiles. Just as was true for $T_{c}$ uniformity, our recipe of a pure TES deposited as first layers on the tile, protected with an oxide film, helps maintain repeatable performance.

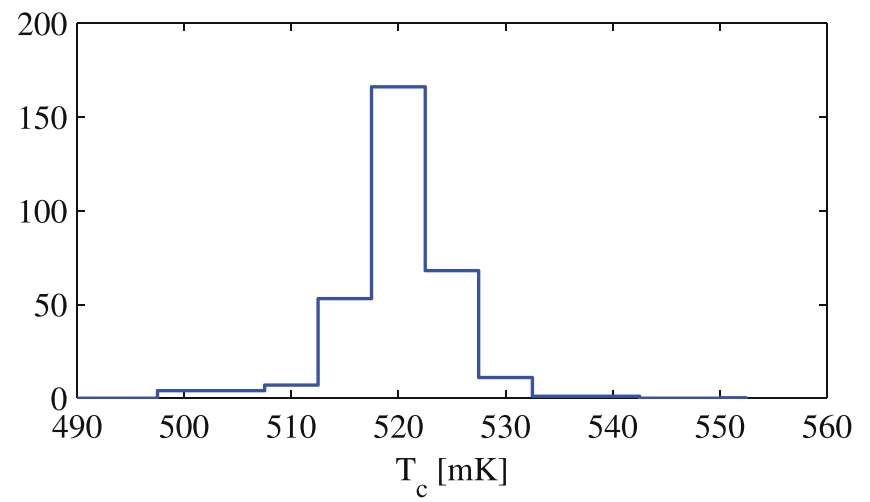

Figure 15. Titanium TES transition temperature for four tiles in BICEP2.

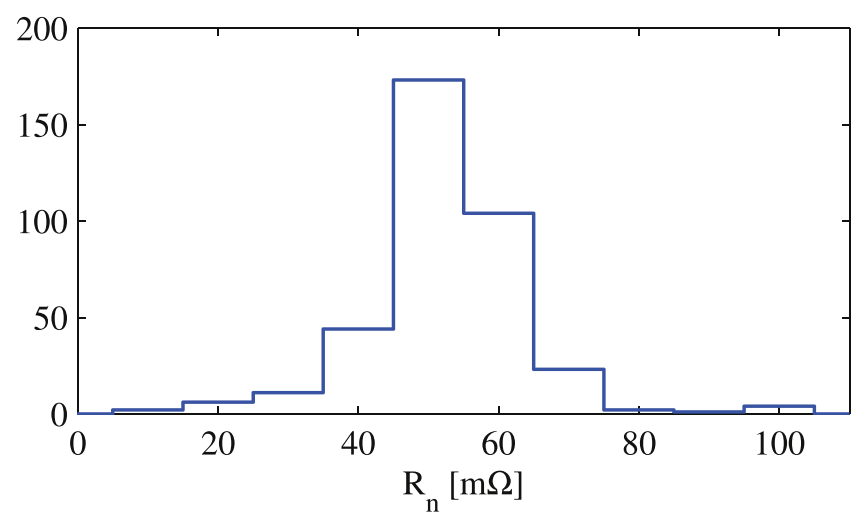

Figure 16. Titanium Normal Resistance for four BICEP2 tiles.

\subsection{Time Constant $\tau$ and Loop Gain $\mathscr{L}$}

The effective thermal time constant of an ideal voltagebiased TES bolometer is given by

$$
\tau=\frac{G / C}{1+\mathscr{L}(V)}
$$

where the intrinsic bolometer time-constant $C / G$ is decreased by the effective loop gain of electrothermal feedback, $\mathscr{L}$. The responsivity of our detectors is the change in measured current for a change in incident optical power, given by

$$
s=\frac{-1}{V_{b}} \frac{\mathscr{L}}{(\mathscr{L}+1)} \frac{1}{(1+i \omega \tau)} .
$$

Both of these expressions experience corrections for nonidealities such as finite shunt resistance. Equations (13) and (14) indicate that a high loop gain $\mathscr{L}$ will increase the speed of the sensor and simplify its responsivity $\left(s \approx-1 / V_{b}\right)$. Operating the bolometers in this limit also maintains a fast detector response (low $\tau$ ), giving rise to an approximately flat detector transfer function for the frequencies of interest, which for our ground-based experiments are $f \lesssim 2 \mathrm{~Hz}$ (Irwin \& Hilton 2005).

In order to maintain stability against electrothermal oscillations, the detectors' thermal bandwidth must not exceed that of the electrical bias circuit. The BICEP2 bias circuit includes a $1.35 \mu \mathrm{H}$ inductor in series with the $\sim 3 \mathrm{~m} \Omega$ bias resistor to avoid aliasing above a roll-off of $R / L \sim 5-6 \mathrm{kHz}$. To limit the TES bandwidth, we deposit thick $\sim 2 \mu \mathrm{m}$ gold onto the BICEP2 detectors that adds an additional $C \sim 0.5 \mathrm{pJ} \mathrm{K}^{-1}$ heat capacity to the bolometer island. The resulting time constants at typical science biases are shown in Figure 17. 

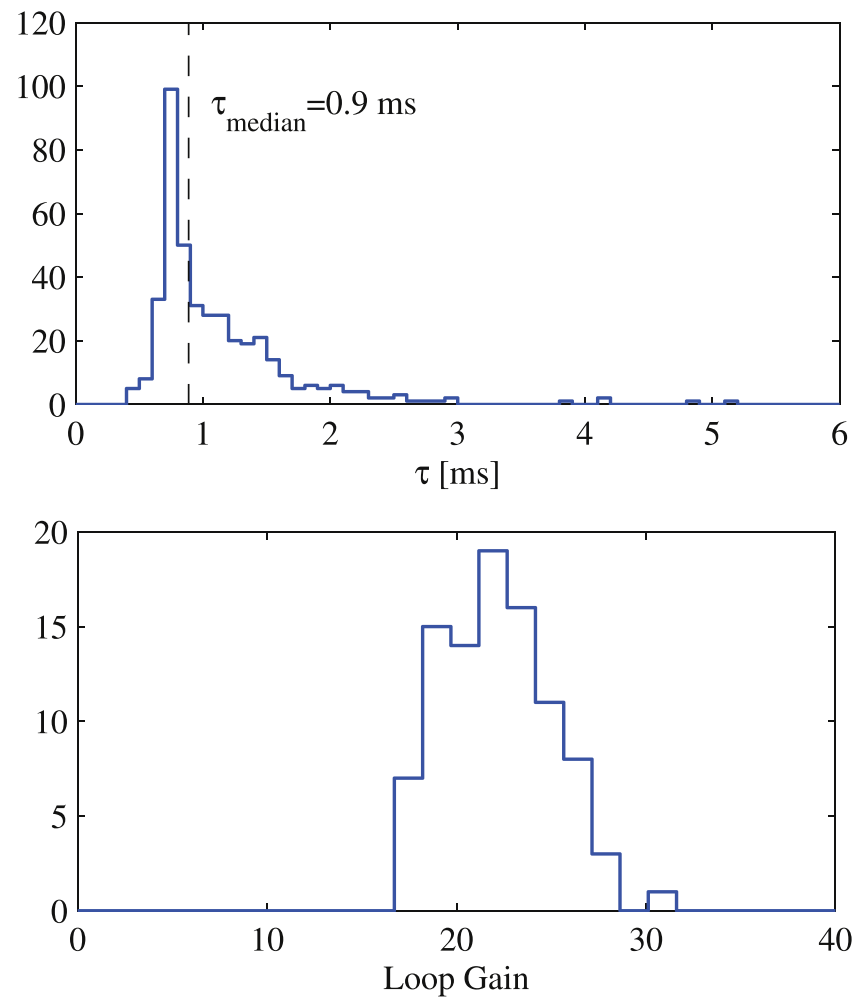

Figure 17. Upper: time constants of BICEP2. These were only carefully measured in transition. Lower: loop gain of SPIDER. We measured time constant at a variety of biases for SPIDER characterization, allowing a proper measurement of loop gain. Both sets of detectors had similar $\tau \sim 0.9 \mathrm{~ms}$ biased time-constants.

SPIDER detectors are intrinsically slower due to their lower leg conductance. It is thus not necessary to add as much gold to the island to ensure stability; typically only $\sim 0.5 \mu \mathrm{m}$ is deposited. Time constants on transition are found to be similar to BICEP2's. For SPIDER detectors we have measured time constants in response to optical square-wave excitations at a range of bias voltages (and thus TES resistances), allowing us to infer that the normal time constants (without loop gain) are $G / C \sim 30 \mathrm{~ms}$ and that loop gains are $\mathscr{L} \sim 20-30$ at $R=0.6 R_{n}$ (Figure 17). BICEP2 TESs should have similar loop gains. We note in passing that fast bolometer time constants are useful for limiting the effect of particle radiation (e.g., cosmic rays) on detector time streams in balloon- and space-borne instruments.

\subsection{Spectral Response}

Our cameras use a series of low-pass filters, both absorptive plastic and reflective metal-mesh, to limit thermal loading on the focal plane and above-band response of our detectors. However, we rely upon the integrated microstrip filters described in Section 3.4 to avoid the atmospheric lines immediately adjacent to our observing bands. Figure 18 shows measured response $S(\nu)$ averaged across a focal plane for three different spectral channels (in three different focal planes), demonstrating that this technology does indeed avoid atmospheric features.

We can summarize the spectral response $S(\nu)$ by comparing it to a top-hat response with the same area and then defining the

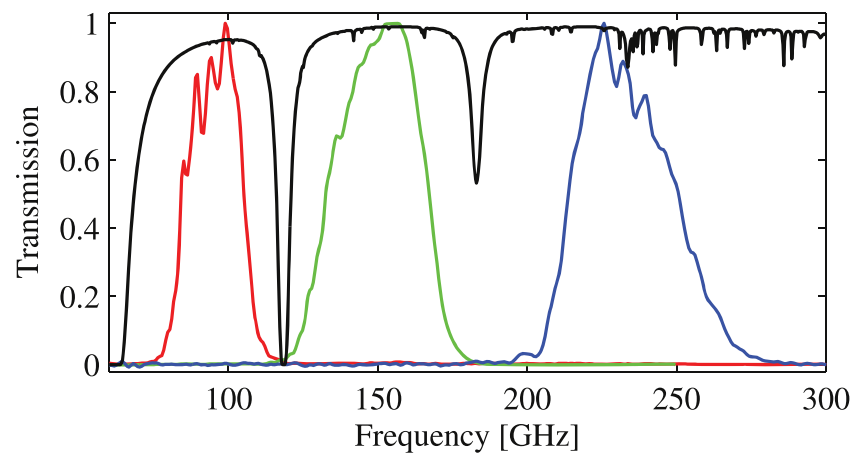

Figure 18. Measured detector spectra for devices designed for $95 \mathrm{GHz}$ (red), $150 \mathrm{GHz}$ (green) and $230 \mathrm{GHz}$ (blue). The data for the $150 \mathrm{GHz}$ are from BICEP2, while the others are from a test cryostat. Winter atmospheric transmission at the South Pole is overlaid in black.

band center as

$$
\langle\nu\rangle=\frac{\int \nu I(\nu) S(\nu) d \nu}{\int I(\nu) S(\nu) d \nu}
$$

and the bandwidth as

$$
\Delta \nu=\frac{\left(\int I(\nu) S(\nu) d \nu\right)^{2}}{\int I^{2}(\nu) S^{2}(\nu) d \nu} .
$$

where $I(\nu)$ is the source spectrum relative to a Rayleigh-Jeans spectrum. The spectral responses of the detectors within a polarization pair must be closely matched to avoid systematic contamination through spectral gain mismatch. In principle, using separate filters for each polarization might induce a mismatch through gradients in material properties. Our design resists this by placing the filter pairs in close physical proximity; in the $150 \mathrm{GHz}$ detectors, the filters are $\sim 5 \mathrm{~mm}$ apart. Furthermore, the filters' magnetic inductance dominates the kinetic inductance; this renders our filters' features particularly robust against variation in $\mathrm{Nb}$ contamination that might adversely impact electrically long resonators (Myers et al. 2005). As shown in Figure 19, our band centers and widths are found to be highly repeatable. We calibrate our responsivity through correlation against the Planck $143 \mathrm{GHz}$ maps. Spectral mismatches between filter pairs would manifest themselves as differences in each detector's absolute calibration (abscal), but the bottom panel of Figure 19 shows that these are less than $3 \%$ of the average responsivity, with a median of $0.5 \%$.

\subsection{Optical Efficiency}

We characterize our end-to-end optical efficiency by biasing onto the aluminum TES, which has a higher $T_{c}$ than Ti, and thus higher saturation power. By comparing the measured response to aperture-filling $77 \mathrm{~K}$ and $300 \mathrm{~K}$ sources to the expected incident power $P_{\text {inc }}=k \Delta T \int f(\nu) d \nu$, we can use the measured spectra to infer efficiency. We routinely obtain total camera optical efficiencies in excess of $30 \%$. For example, the BICEP2 efficiencies are shown in Figure 20. These figures are for the total camera efficiency, which includes losses other than the detectors. Measurements of response of early engineeringgrade detectors to an internal cold load, which exclude spillover loss and loss in our filter stack, suggest that the raw detector efficiency should be nearly 70\% (Orlando et al. 2009). 

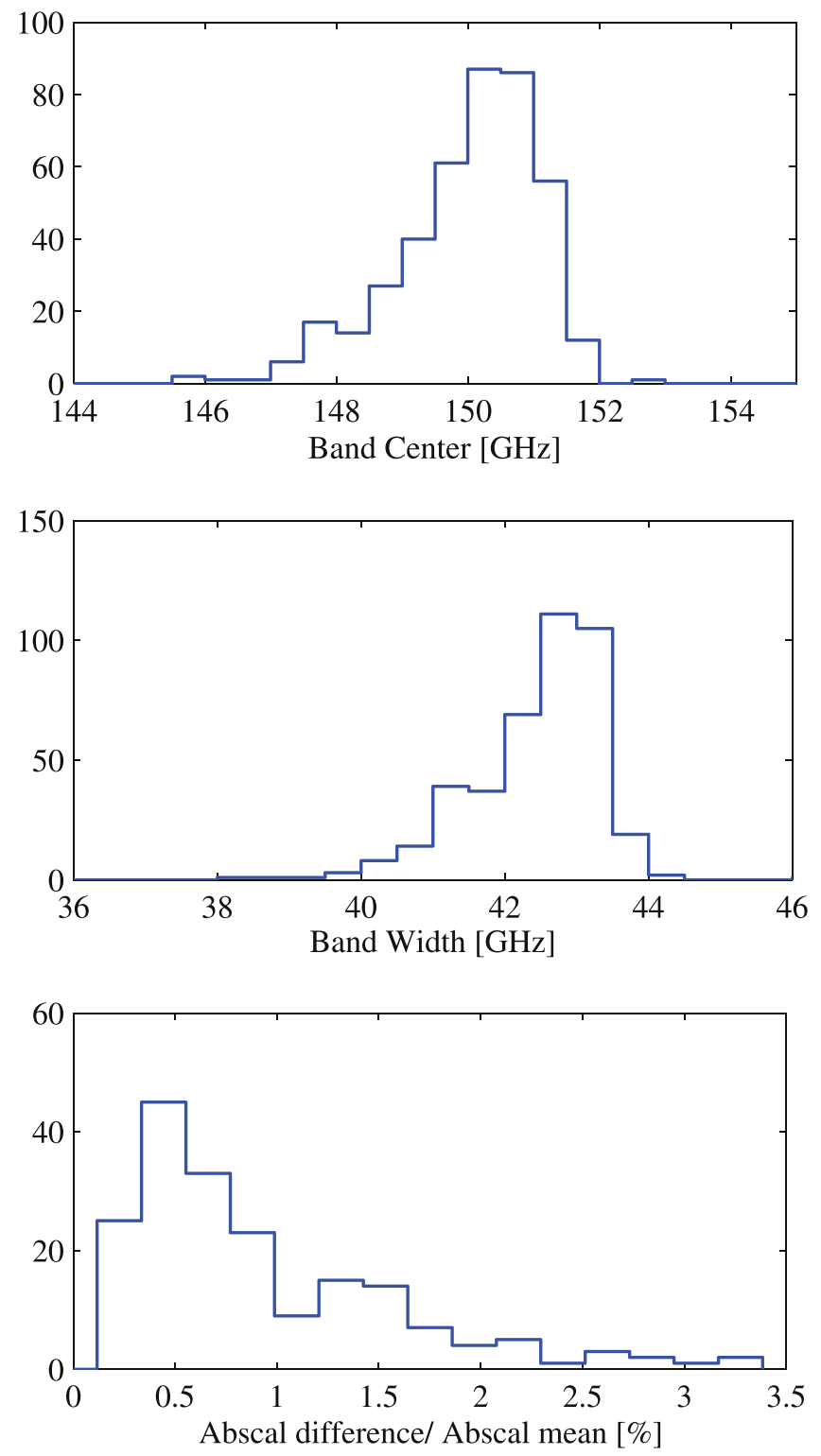

Figure 19. Spectral response of detectors in the BICEP 2 camera. Upper: histogram of the band centers. Middle: histogram of band widths. Lower: histogram of absolute calibration (abscal) mismatch, where difference and mean are computed between polarization pairs. Calibration is through crosscorrelation against Plank $143 \mathrm{GHz}$.

\section{SENSITIVITY}

The array design described above was first deployed for astronomical measurements in 2009 as part of BICEP2, a groundbased CMB polarimeter. We have since fabricated dozens of deployment-grade arrays at 95 and $150 \mathrm{GHz}$ for use in the terrestrial Keck Array and balloon-borne SPIDER instruments. These programs have yielded extensive data on the real-world performance of this technology, as well as demonstrating its adaptability to different optical loads.

\subsection{Noise}

The theory of noise in TES bolometers is by now well developed (Irwin \& Hilton 2005). Major contributions typically include photon noise, phonon noise (thermal fluctuation noise), Johnson noise from the TES and shunt resistances, and

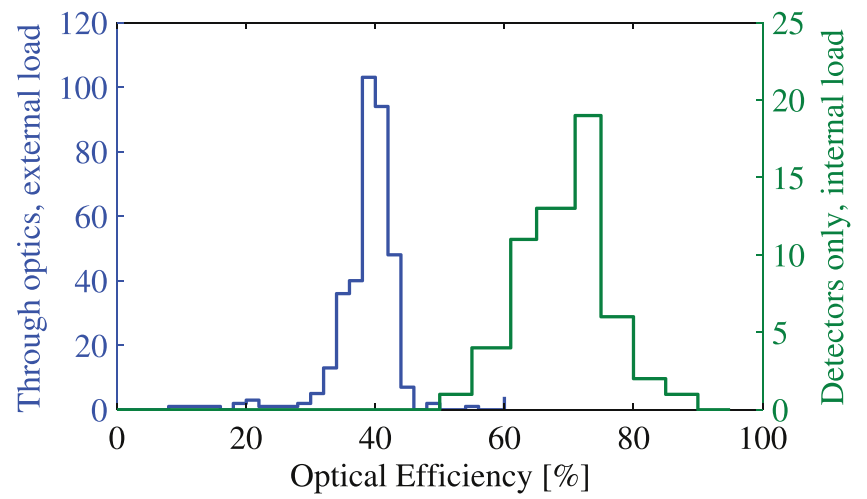

Figure 20. Optical Efficiencies of BICEP2 detectors. Blue curves (left-axis) are end-to-end receiver efficiency through all optics; green curves (right-axis) are raw detector efficiencies for a single test-tile from an engineering-grad test focal plane, in response to an internal cold-load.

amplifier noise. In many cases TESs also exhibit varying degrees of "excess" noise beyond that predicted by simplified models. In multiplexed systems, noise performance also depends upon the relationship between the noise levels and detector and readout bandwidths, since poor choices can lead to substantial noise aliasing (Battistelli et al. 2008, and Stiehl et al. 2011). We find that the detectors" measured noise is well reproduced by a simple model incorporating modest excess TES noise, and we have successfully operated the bolometers in configurations with little aliasing penalty.

In order to characterize the noise performance, we have measured noise spectra for the BICEP2 and Keck Array detectors using un-multiplexed data digitized at $400 \mathrm{kHz}$. Although the frequencies corresponding to degree-scale anisotropies is less than $2.6 \mathrm{~Hz}$ (where $\ell \sim 500$ ) for the BICEP2 and Keck Array scan strategy, these measurements allow us to observe device performance near the typical multiplexing frequency of $25 \mathrm{kHz}$ and thus model noise aliasing due to the readout. These measurements, adjusted for expected aliasing, are in good agreement with noise measured in the science-mode multiplexer configuration.

The right panel of Figure 21 compares the measured noise for a representative optically active "light" detector from the Keck Array to its various modeled components. Photon noise dominates at the low frequencies of interest in the light pixels for sky observations. The photon noise equivalent power (NEP) can be expressed as a sum of Bose and shot noise contributions:

$$
\mathrm{NEP}_{\text {photon }}^{2}=2 h \nu Q_{\text {load }}+\frac{2 Q_{\text {load }}^{2}}{\Delta \nu},
$$

where $\nu$ is the band center, $\Delta \nu$ is the bandwidth, and $Q_{\text {load }}$ is the optical loading. The next-largest contribution to the noise at low frequencies is the thermal fluctuation (phonon) noise across the SiN isolation legs, given by Equation (10). For the optically inactive "dark pixels," such as that in the left panel of Figure 21, photon noise is present through $0.05 \mathrm{pW}$ of direct loading (not through the antenna), but is subdominant to phonon noise. All other modeled contributions, including the TES Johnson and excess noise, the shunt resistor noise, and the cold and warm amplifiers, are negligible at low frequencies.

Below $7 \mathrm{~Hz}$, atmospheric fluctuations begin to dominate the light pixels, an effect which is absent in the dark spectra. We find that this common mode unpolarized signal subtracts well 


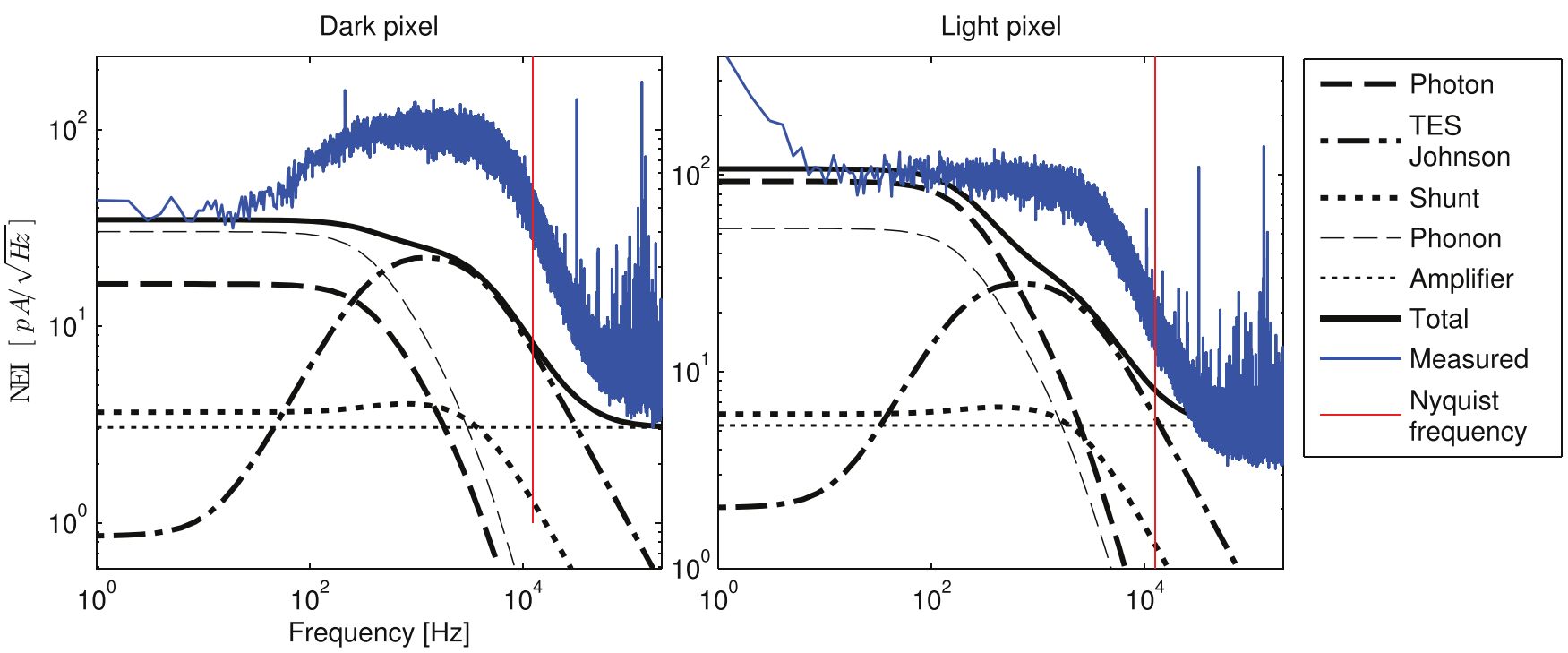

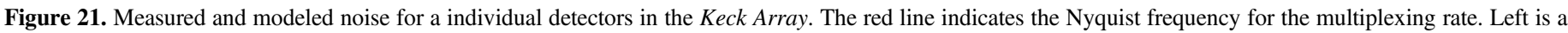

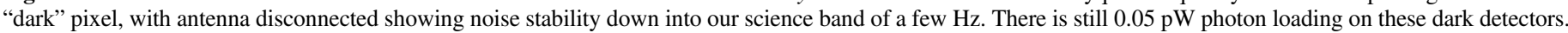

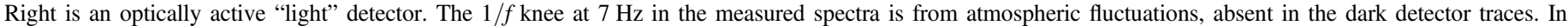

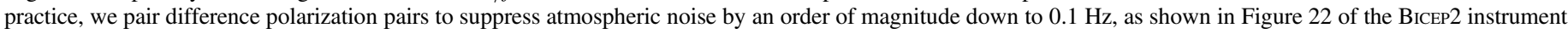

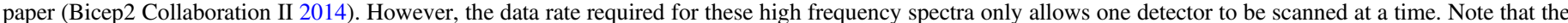

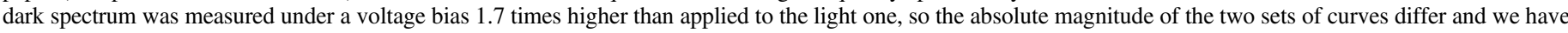

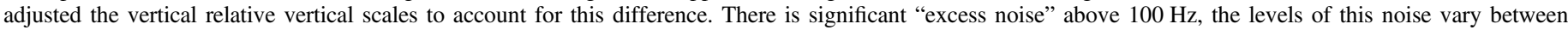
detectors. The difference in this excess noise between the light and dark pixels is consistent with this variance.

down to below $0.1 \mathrm{~Hz}$ when we polarization-pair difference, as shown the Figure 22 of the BICEP2 instrument paper (Bicep2 Collaboration II 2014).

At frequencies of $\approx 1 \mathrm{kHz}$ the TES excess noise starts to contribute significantly. The TES excess noise, as described in Gildemeister et al. (2001) tends to be proportional to the TES transition steepness $\beta=\left.(R / I)(\partial I / \partial R)\right|_{T}$, which for these detectors is higher at lower resistances. The excess noise varies between fabrication batches, although the detector in Figure 21 has a relatively low amount. The excess noise should have little contribution to an experiment's overall noise level as long as it is not aliased. BICEP2 and the Keck Array avoid such aliasing by multiplexing at $25 \mathrm{kHz}$ and biasing at relatively high resistances on detectors that have large excess noise components. SPIDER cannot multiplex as quickly due to long cable lengths, so we use a lower TES resistance $\left(R_{n} \sim 30 \mathrm{~m} \Omega\right)$ and higher Nyquist inductance $(2.0 \mu \mathrm{H})$ to limit aliasing of excess noise.

The noise contributions for BICEP2 and the Keck Array at low frequencies and under winter atmospheric loading conditions are broken down in Table 3 for the observing year 2012. The photon noise is of absorbed power only to aid the comparison between models and measurements without adding an extra factor of optical efficiency. The last line of the table shows total noise equivalent temperature (NET) computed from jackknife maps, which necessarily references noise of the incident photons and accounts for the optical efficiency of the cameras.

\subsection{Performance of Ground-based Designs}

We have measured the devices' performance on the sky during lengthy BICEP2 and Keck Array observation campaigns. The sensitivity of the experiment can be measured using the timestream noise between 0.1 and $1 \mathrm{~Hz}$, after calibrating to the CMB. BICEP2 had a NET of $16 \mu \mathrm{K}_{\mathrm{CMB}} \sqrt{\mathrm{s}}$. The larger detector

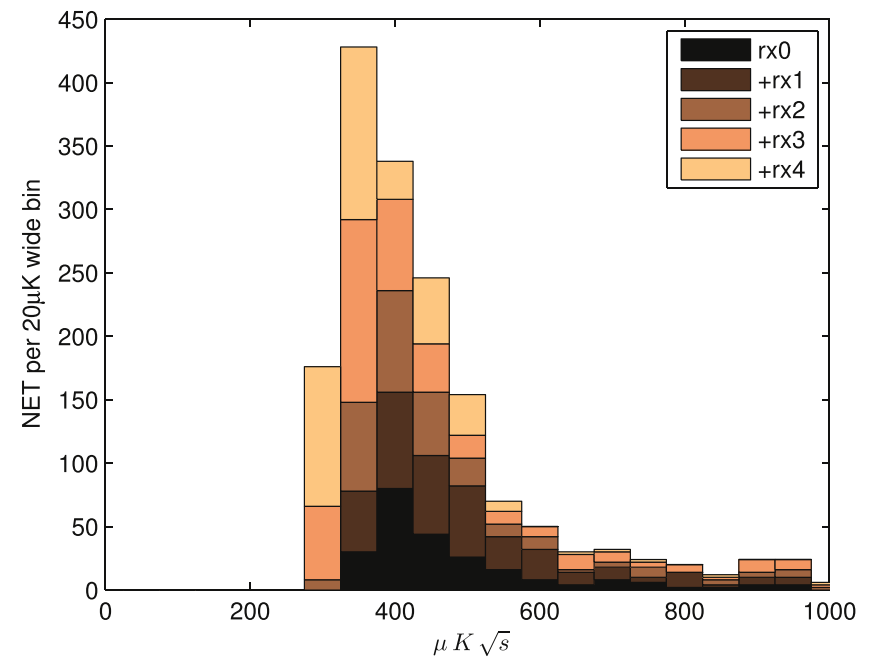

Figure 22. NET per detector histogram for the Keck Array in 2013.

complement of the Keck Array achieved 11.5 and $9 \mu \mathrm{K}_{\mathrm{CMB}} \sqrt{\mathrm{s}}$ in 2012 and 2013, respectively, also at $150 \mathrm{GHz}$. Figure 22 shows the distribution of per-detector sensitivity for the Keck Array in 2013.

Another measure of instrument performance is the total map depth achieved, defined as the noise within a map pixel of a specific size. This is a function of instrumental sensitivity, scan strategy, and observing time. Over three seasons of observing, BICEP2 achieved an rms noise of $\mathrm{NEQ}=87 \mathrm{nK}$ deg $(5.2$ $\mu \mathrm{K}$ arcmin; Bicep2 Collaboration I 2014, Bicep2 Collaboration II 2014). Over two years, the five Keck Array $150 \mathrm{GHz}$ cameras achieved $\quad \mathrm{NEQ}=74 \mathrm{nK}$ deg $\quad(4.4 \mu \mathrm{K}$ arcmin $) . \quad$ Combining BICEP2 and Keck Array and averaging across the entire effective area 400 square degree field results in rms noise of $2 \mathrm{nK}$. 
Table 3

Summary of Detector Noise are Low Frequencies for BICEP2 and Keck Array

\begin{tabular}{lccccc}
\hline \hline Receiver & BICEP2 & Keck Array Rx0 & Rx1 & Rx2 & Rx3 \\
\hline Absorbed Photon Noise $[\mathrm{aW} / \sqrt{\mathrm{Hz}}]$ & 41 & 33 & 32 & 34 & 27 \\
Phonon Noise $[\mathrm{aW} / \sqrt{\mathrm{Hz}}]$ & 27 & 24 & 20 & 24 & 14 \\
TES Johnson Noise $[\mathrm{aW} / \sqrt{\mathrm{Hz}}]$ & 0.5 & 0.8 & 0.5 & 0.8 & 0.4 \\
Amplifier Noise $[\mathrm{aW} / \sqrt{\mathrm{Hz}}]$ & 2.0 & 2.0 & 2.0 & 2.4 & 0.6 \\
Total Noise + Aliased at $25 \mathrm{kHz}[\mathrm{aW} / \sqrt{\mathrm{Hz}}]$ & 56 & 46 & 39 & 48 & 3.0 \\
per-detector NET from maps $\left[\mu \mathrm{K}_{\mathrm{CMB}} \sqrt{\mathrm{s}}\right]$ & 305 & 316 & 351 & 317 & 370 \\
\hline
\end{tabular}

\section{CONCLUSIONS AND FUTURE WORK}

We have demonstrated our novel detectors' performance through 18 camera-years of observations in the BICEP2/Keck Array program, including a detection reported in 2014 of degree-scale B-mode anisotropy (Bicep2 Collaboration I 2014). This paper has described the design principles, challenges, fabrication techniques and our characterization/screening program that made these deployments possible. This program has continued through the recent, successful 2015 SPIDER flight and higher frequency upgrades to Keck Array.

We have also deployed BICEP3 in the current (2014-2015) Antarctic summer season, which has $115295 \mathrm{GHz}$ detectors in a single camera. Once all the detectors are installed in the 2015-2016 season, this instrument will support $256095 \mathrm{GHz}$ detectors. We package these detectors in individual modules that efficiently fill the focal plane and we illuminate the detectors' antennas with non-uniform Gaussian tapered slot illuminations to reduce spillover onto the camera's stop. These recent modifications will be the subject of a future paper.

Lastly, our detector technology allows for multi-color focal planes where different color channels are co-located on the focal plane. Most competing dual-band detector technologies use a single common aperture for all color channels and thus there is a reduction in per-detector efficiency due to aperture spillover (O'Brient et al. 2013). Planar antenna arrays allow each color to have a custom aperture, thus more efficiently using of both focal plane real estate and limited readout capacity. These are under active development for BICEP3 further upgrades to the BICEP/Keck Array program, and for future SPIDER flights.

The development of antenna-coupled detector technology was supported by the JPL Research and Technology Development Fund and grants 06-ARPA206-0040 and 10-SAT10-0017 from the NASA APRA and SAT programs. The development and testing of focal planes were supported by the Gordon and Betty Moore Foundation at Caltech. Readout electronics were supported by a Canada Foundation for Innovation grant to UBC. The receiver development was supported in part by a grant from the W. M. Keck Foundation. BICEP2 was supported by the US National Science Foundation under grants ANT0742818 and ANT-1044978 (Caltech/Harvard) and ANT0742592 and ANT-1110087 (Chicago/Minnesota). Some computations in this paper were run on the Odyssey cluster supported by the FAS Science Division Research Computing Group at Harvard University. We thank Warren Holmes and
Matthew Kenyon for discussions on thermal conductivity. Tireless administrative support was provided by Irene Coyle and Kathy Deniston.

\section{REFERENCES}

Battistelli, E., Amiri, M., Burger, B., et al. 2008, JLTP, 151, 908 Bicep2 Collaboration I 2014, PhRvL, 112, 241101

Bicep2 Collaboration II 2014, ApJ, 792, 62

Bicep2 Collaboration III 2015, arXiv:1502.00608

Bicep2 Collaboration and Keck Array Collaborations IV 2015, ApJ, 806, 206

Bodharamik, P., Besser, L., \& Newcomb, R. 1971, IEEE Transactions on Circuit Theory, 18, 610

Corp, C. S. T. 2009a, CST Microwave Suite User manual (CST-AG)

Corp, S. 2009b, Sonnet User manual v. 12.52 (Sonnet)

Das, N., \& Pozar, D. M. 1987, ITMTT, 35, 326

de Korte, P. A. J., Beyer, J., Deiker, S., et al. 2003, RScI, 74, 3807

Duband, L., \& Collaudin, B. 1999, Cryo, 39, 659

Fabrega, L., Fernandez-Martinez, I., Gil, O., et al. 2009, ITAS, 19, 460

Fraisse, A. A., Ade, P. A. R., Amiri, M., et al. 2013, JCAP, 4, 47

Galbraith, C., \& Rebeiz, G. 2008, ITMTT, 56, 1675

Gildemeister, J. M., Lee, A. T., \& Richards, P. L. 1999, ApPhL, 74, 868

Gildemeister, J. M., Lee, A. T., \& Richards, P. L. 2001, ApOpt, 40, 6229

Gudmundsson, J. E., Ade, P. A. R., Amiri, M., et al. 2015, arXiv:1506.06953

Hammerstad, E., \& Jensen, O. 1980, IEEE MTT-S International, Microwave Symposium, 407

Holmes, W., Gildemeister, J. M., Richards, P. L., \& Kotsubo, V. 1998, ApPhL, 72,2250

Holmes, W. A. 1998, PhD thesis, Univ. California

Irwin, K., \& Hilton, G. 2005, in Cryogenic Particle Detection, ed. C. E. Ascheron, H. J. Koelsch, \& W. Skolaut (Heidlberg: Springer), 63

Kamionkowski, M., Kosowsky, A., \& Stebbins, A. 1997, PhRvL, 78, 2058

Kaplinghat, M., Knox, L., \& Song, Y.-S. 2003, PhRvL, 91, 241301

Kernasovskiy, S., Ade, P. A. R., Aikin, R. W., et al. 2012, Proc. SPIE, 8452, 1

Kominami, M., Pozar, D. M., \& Schaubert, D. 1985, ITAP, 33, 600

Kuo, C. L., Bock, J. J., Bonetti, J. A., et al. 2008, Proc. SPIE, 7020

Martin, D., \& Puplett, E. 1970, InfPh, 10, 105

Mather, J. C. 1982, ApOpt, 21, 1125

Myers, M. J., Holzapfel, W., Lee, A. T., et al. 2005, ApPhL, 86, 114103

O'Brient, R., Ade, P., Arnold, K., et al. 2013, ApPhL, 102, 063506

O'Brient, R., Ade, P. A. R., Ahmed, Z., et al. 2012, Proc. SPIE, 8452, $84521 \mathrm{G}$

Orlando, A., Aikin, R. W., Amiri, M., et al. 2009, in AIP Conf. Proc. 1185, The Thirteenth International Wokshop on Low Temperature Detectors ed. B. Young, B. Cabrera, \& A. Miller (Melville, NY: AIP: AIP), 471

Rego, L. G. C., \& Kirczenow, G. 1998, PhRvL, 81, 232

Schwab, K., Henriksen, E. A., Worlock, J. M., \& Roukes, M. L. 2000, Natur, 404, 974

Stiehl, G., Cho, H.-M., Hilton, G., et al. 2011, ITAS, 21, 298

Takahashi, Y. D., Ade, P. A. R., Barkats, D., et al. 2010, ApJ, 711, 1141

Tinkham, M. 1996, Introduction to Superconductivity (2nd ed.; New York: McGraw Hill)

Turner, A. D., Bock, J. J., Beeman, J. W., et al. 2001, ApOpt, 40, 4921

Williams, K., Gupta, K., \& Wasilik, M. 2003, JMemS, 12, 761

Zaldarriaga, M., \& Seljak, U. 1997, PhRvD, 55, 1830

Ziman, J. 1963, Electrons and Phonons (Oxford: Oxford Univ. Press) 